\title{
On the Determination of Groundwater Level using Temporal and Spatial Parameters: Advanced Machine Learning Methods
}

\section{Amirhossein Najafabadipour ( $\square$ najafabadipour@eng.uk.ac.ir)}

Shahid Bahonar University Faculty of Engineering https://orcid.org/0000-0003-3411-8122

Gholamreza Kamali

Shahid Bahonar University Faculty of Engineering

Hossein Nezamabadi-pour

Shahid Bahonar University Faculty of Engineering

\section{Research Article}

Keywords: CF, Committee machine intelligent system, GR, Groundwater Level, MLP, RBF

Posted Date: April 8th, 2021

DOI: https://doi.org/10.21203/rs.3.rs-234142/v1

License: (1) This work is licensed under a Creative Commons Attribution 4.0 International License.

Read Full License 


\section{On the Determination of Groundwater Level using Temporal and Spatial \\ 2 Parameters: Advanced Machine Learning Methods}

4 Amirhossein Najafabadipour ${ }^{1}$. Gholamreza Kamali ${ }^{1}$. Hossein Nezamabadi-pour ${ }^{2}$

6 Abstract

7 Prediction of groundwater level is a useful tool for managing groundwater resources in the mining

8 area. Water resources management requires identifying potential periods for groundwater drainage

9 to prevent groundwater from entering the mine pit and reduce high costs. For this purpose, four 10 multilayer perceptron (MLP) neural network models and four cascade forward (CF) neural network 11 models optimized with Bayesian Regularization (BR), Levenberg-Marquardt (LM), Resilient

12 Backpropagation (RB), and Scaled Conjugate Gradient (SCG), as well as a radial basis function 13 (RBF) neural network model and a generalized regression (GR) neural network model were 14 developed to predict groundwater level using 1377 data point. This data set includes 12 spatial 15 parameters divided into two categories of sediments and bedrock, and besides, 6 time series 16 parameters have been used. Also, to determine the best models and combine them, 165 extra
Amirhossein Najafabadipour
najafabadipour@eng.uk.ac.ir
${ }^{1}$ Department of Mining Engineering, Shahid Bahonar University of Kerman, Kerman, Iran
${ }^{2}$ Department of Electrical Engineering, Shahid Bahonar University of Kerman, Kerman, Iran 
17 validation data points have been used. After identifying the best models from the three candidate

18 models with lower average absolute relative error (AARE) value, the committee machine

19 intelligence system (CMIS) model has been developed. The proposed CMIS model predicts

20 groundwater level data with high accuracy with an AARE value of less than $0.11 \%$. Also, the

21 proposed model was compared with ten other models through graphical and statistical error

22 analysis. The results show that the developed CMIS model performs better than other existing

23 models in terms of precision and validity range. The relevancy factor indicates that the electrical

24 resistivity of sediments had the highest effect on the groundwater level. Eventually, the quality of

25 the data used was investigated both statistically and graphically, and the results show satisfactory

26 reliability of the data used.

27 Keywords: CF, Committee machine intelligent system, GR, Groundwater Level, MLP, RBF

\section{1. Introduction}

29 Groundwater is a natural resource that has costly adverse effects on mining operations (Brawner 30 1986). Due to the increased depth of mining, excavation may be done below the water table, which

31 leads to the movement of water towards mining works. Too much water entering the mining

32 environment may delay the project or impede production, in addition to causing environmental and

33 safety problems (Singh and Atkins 1985). Increased equipment failure, lack of access to part of the

34 mining area, increased use of explosives, loading problems, unsafe working conditions, and a

35 damaging effect on pit slope stability are among the undesirable impacts of groundwater entering

36 the mining environment. Therefore, to overcome these problems, it is necessary to develop an

37 efficient dewatering system that prediction of groundwater level can contribute significantly to this 
38 design. Various classical and modern methods can be used to model and predict groundwater 39 fluctuations.

40 For classic physical-based modeling, the study area's exact characteristics and boundary conditions

41 must be accurately identified, and this model is based on some assumptions. Therefore, a physical-

42 based model is not proper for all hydrogeological situation. The numerical models are extensively

43 used to simulate the quantity and quality of groundwater (Xu et al. 2011). Numerical modeling of

44 groundwater by this model (i.e., MODFLOW) requires some input parameters; hence preparing

45 proper values for these parameters is a time-consuming and costly activity. To control limitations, 46 soft computing techniques are a valid alternative to providing more precision with less

47 computational time to predict groundwater levels (Golestani Kermani et al. 2019).

48 To predict groundwater levels and the complexity of subsurface conditions, machine learning 49 methods based on nonlinear dependence can be used without deep knowledge of basic physical 50 parameters (Parisouj et al. 2020). In recent years, artificial intelligence (AI) methods have been 51 widely used to predict water system variables due to their high ability to learn complex 52 mathematical relationships between output and prediction variables. One of the most common 53 machine learning algorithms used to predict groundwater level is the artificial neural network 54 (ANN) (Santos Finck and Correa Pedrollo 2021). multilayer perceptron (MLP), Cascade forward 55 (CF), Radial basis function (RBF), General regression (GR) and, committee machine intelligence 56 system (CMIS) are the most widely used artificial neural network methods based on their different 57 architectures. Kouziokas et al. (2018) used MLP to predict groundwater levels in Montgomery, 58 Pennsylvania. Hayder et al. (2020) modeled river flow using optimized CF and MLP in the Kelantan 59 River in Malaysia. Hong et al. (2020) predicted trihalomethanes levels in tap water using RBF and 60 gray relational analysis (GRA). Pal and Chakrabarty (2020) have evaluated GR neural network 
61 models in simulating the groundwater contaminant transport. Malekpour and Mohammad Rezapour

62 Tabari (2020) have implemented the supervised intelligence committee machine method to predict

63 reservoir water level variation for the design and operation of dams. In addition to different methods

64 for modeling and predicting groundwater levels, different spatial and temporal data can affect

65 groundwater levels (Rajaee et al. 2019). Most studies have used spatial or temporal parameters to

66 predict groundwater levels using machine learning, but both spatial and temporal parameters affect

67 groundwater levels.

68 The main purpose of this research is to develop and compare widely used and accurate machine

69 learning methods to predict groundwater levels using both spatial and temporal parameters. For this

70 purpose, 1542 data points including 12 spatial parameters and 6 time series parameters were used.

71 Out of 1377 data have been used to create different networks, and to evaluate the performance of

72 these developed models, 165 extra validation data have been used. Four multilayer perceptron

73 neural network (MLP) models and four cascade forward neural network (CF) models were then

74 developed using four different optimized Bayesian Regularization (BR), Levenberg-Marquardt

75 (LM), Resilient Backpropagation (RB), and Scaled Conjugate Gradient (SCG). Also, the radial

76 basis function neural network (RBF) and general regression neural network (GR) methods have

77 been used to model the groundwater level. Although CF and GR models have been used for water

78 resource management, these models have not yet been developed for the prediction of groundwater

79 level. After developing models, a committee machine intelligent system (CMIS) is combined of

80 three candidate models with the least error. The validity of the proposed CMIS is evaluated through

81 statistical and graphical error analysis. The innovation of this research, in addition to identifying

82 the relevancy factor of the data relative to the groundwater level, is also identifying outliers. 
83 The rest of this research is organized as follows: Section 2 provides information on the study area

84 and the data used; Sections 3 and 4 describe the developed models and optimization techniques,

85 respectively; Section 5 outlines the results and discussion of this research, and section 6 presents

86 the conclusions of this research.

\section{2. Experimental Data}

88 Gol Gohar iron ore deposit, one of the most popular pivot points of the mining industry in the

89 Middle East, with six separate anomalies and a reservoir of about 1200 million tons, is located in

90 an area with a length of approximately $10 \mathrm{~km}$ and nearly $4 \mathrm{~km}$ in width. In anomaly No.3 (Gohar

91 Zamin Iron Ore Mine), groundwater enters the pit, and also water permeates through the alluvium

92 of the pit's stairs. One of the probable factors of going groundwater inflow into Gohar Zamin Iron

93 Ore Mine is the Kheyrabad plain with alluvial sediments situated in the northeast of the mine at a

94 distance of $15 \mathrm{~km}$ (Fig. 1.). Around the Gohar Zamin Iron Ore Mine, water pumping wells are

95 located around anomaly No. 1, which is considered as a discharge area.

96 To estimate the spatial and temporal groundwater level as the target and output of the neural

97 network, two sets of spatial and temporal data have been used as the input of the neural network.

98 The input spatial data set includes five piezometer data around the Gohar Zamin Iron Ore Mine.

99 Besides, the input temporal data consists of the time series of parameters affecting the groundwater

100 level in the period of March 21, 2019, to July 2, 2020. Because both sediments and bedrock affect

101 the groundwater level, the spatial data input to the neural network is divided into two categories of

102 bedrock and sediment parameters. In Table 1, input spatial data to the neural network including

103 latitude and longitude of piezometers, hydraulic conductivity of sediments and bedrock, effective

104 porosity of sediments and bedrock, the electrical resistivity of sediments and bedrock, the 
105 piezometers surface level, bedrock height, Depth of sediments and the presence or absence of faults

106 are shown. The input temporal data to the neural network include day, month, year, drainage

107 volume, evaporation, and precipitation, which in Table 2, statistical explanation of these parameters

108 are shown.

\section{3. Models}

110

\subsection{Multilayer perceptron neural network (MLP)}

111 Artificial neural networks (ANN) as a useful computational intelligence built on analogy with

112 human information processing systems widely used in distributed processing systems (Hebb 1949).

113 Although the structures of the artificial neural network (ANN) are very varied, multilayer

114 perceptron (MLP) is still one of the most dominant as well as the most extensive structures of the

115 artificial neural network. The MLP shown by Cybenko's theorem (1989) is a universal function

116 approximation used to create mathematical models using regression analysis. With training on

117 observation data, the network can learn specific features hidden in the collected data samples and

118 even generalize what it has learned. MLP networks have a multilayered structure, and the first layer

119 is the input data to the model, the last layer is the output data of the model, and the layers between

120 the input and output are called hidden layers. The number of neurons in the input layer is the same

121 as the input variables, the number of outputs is usually the same as the output parameter, and the

122 hidden layers are responsible for the internal appearance of the relationship between the model

123 inputs and the desired output. The value of each neuron in the hidden layer or the output layer is

124 the sum of each neuron in the previous layer, multiplied in a particular weight for that neuron, and

125 summed with bias, and passed from an activation function. The following is a summary of some of

126 the common activation functions used for hidden and output layers. 


$$
\begin{gathered}
\text { Linear=Purelin: } f(x)=x \\
\text { Logsig=Sigmoid: } f(x)=\frac{1}{1+e^{-x}} \\
\text { Tansig=Tanh: } f(x)=\frac{e^{x}-e^{-x}}{e^{x}+e^{-x}} \\
\text { Elliot } 2 \text { Symmetric Sigmoid: } f(x)=\frac{x^{2} \operatorname{SIGN}(x)}{1+x^{2}}
\end{gathered}
$$

127 The output of an MLP model with two hidden layers whose activation functions for these two layers 128 are Logsig and Tansig, and Purlin activation function for the output layer are as follows (Hemmati129 Sarapardeh et al. 2018):

$$
\text { Output }=\text { Purelin }\left(w_{3} \times\left(\text { Tan sig }\left(w_{2} \times\left(\log \operatorname{sig}(x)+b_{1}\right)\right)+b_{2}\right)+b_{3}\right)
$$

130 Where $w_{1}$ and $b_{1}$ are the weight matrixes and the bias vectors of the first hidden layers, $w_{2}$ and $b_{2}$ 131 are the weight matrixes and the bias vectors of the second hidden layers, and $w_{3}$ and $b_{3}$ are the 132 weight matrixes and the bias vectors of the output layers.

\section{$133 \quad 3.2$ Cascade forward neural network (CF)}

134 There is a direct relationship between input and output in the perceptron connection, whereas in the 135 feedforward neural network connection, there is an indirect relationship between input and output, 136 which is a hidden layer through a nonlinear activation function (Fahlman and Lebiere 1989). If the 137 connection form is combined in a multilayer network and perceptron, the network can be formed 138 with a direct connection and the indirect connection between the input layer and the output layer 139 (Hayder et al. 2020). The network formed of this connection pattern is called cascade forward neural 140 network (CF), and the equations of this model can be written as follows:

$$
y=\sum_{i=1}^{n} f^{i} w_{i}^{i} x_{i}+f^{o}\left(w^{b}+\sum_{j=1}^{k} w_{j}^{o} f^{h}\left(w_{j}^{b}+\sum_{i=1}^{n} w_{j i}^{h} x_{i}\right)\right)
$$


141 Where $\mathrm{f}^{\mathrm{i}}$ is the activation function between the input layer and the output layer, $\mathrm{w}_{\mathrm{i}}^{\mathrm{i}}$ is the weight

142 between the input layer and the output layer, $w^{b}$ is the weight from bias to output, $w_{j}^{b}$ is the weight

143 from bias to hidden layer, and $\mathrm{f}^{\mathrm{h}}$ is the activation function of each neuron in the hidden layer.

144 Fig. 2. shows the CF architecture where there is a direct relationship between input and output.

145 Because the optimization algorithm used for training has influential impress in the efficiency of the

146 MLP and CF model, in this research, four important Bayesian Regularization (BR), Levenberg-

147 Marquardt (LM), Resilient Backpropagation (RB), and Scaled Conjugate Gradient (SCG)

148 optimization algorithms have been used.

$149 \quad$ 3.3 Radial basis function neural network (RBF)

150 Radial basis function neural network $(\mathrm{RBF})$ is one of the powerful feedforward neural networks

151 that use performance approximation theory in solving regression problems. Broomhead and Lowe

152 (1988), based on adaptive function interpolation, introduced an approach to local functional

153 approximation. In general, an RBF neural network has a three-layer feedforward structure in which

154 the input layer and the output layer are connected through a hidden layer. The principal part of the

155 RBF neural network is the hidden layer, which transmits data from the input space to the hidden

156 space with higher dimensions (Hemmati-Sarapardeh et al. 2018). Each point in the hidden layer

157 with a particular radius is located at a given space, that in each neuron, the distance between the

158 input vector and its center is calculated. Euclidean distance is used to measure the distance between

159 centers and inputs, which is calculated from the following equation:

$$
r_{j}=\sqrt{\sum_{i=1}^{p}\left(x_{i}-c_{i j}\right)^{2}}
$$


160 For a model with ten input variables, $\mathrm{P}=10$. To transfer the Euclidean distance from each neuron in 161 the hidden layer to the output, a radial basis function has been used. The most common radial basis

162 function is the Gaussian, which is obtained from the following interface:

$$
\text { Gaussian: } \varnothing(r)=\exp \left(\frac{r^{2}}{2 \sigma^{2}}\right)
$$

163 Due to its smoother and flexible behavior, the Gaussian function has been utilized as the activation

164 function in this research. When $x=c_{j}, \emptyset(r)$ is maximum, and as $r$ increases, the $\emptyset(r)$ decreases. 165 when $|r| \rightarrow \infty . \varnothing(r) \rightarrow 0 . \sigma$ is the spreading coefficient of the Gaussian function, which is defined

166 experimentally (Meshram et al. 2020). The model output is estimated from the following equation:

$$
y_{k}=\sum_{j=1}^{N} \omega_{j} \emptyset_{k j}\left(\left\|x_{k}-c_{j}\right\|\right) j=1 \ldots . . N \text { and } k=1 \ldots . M
$$

167 Where $\omega$ shows connection weight, $N$ is the number of neurons in the hidden layer, c denotes the 168 center, $(\|\mathrm{x}-\mathrm{c}\|)$ is Euclidean distance between the center of the radial function and the input data 169 (Menad et al. 2019).

\subsection{Generalized regression neural network (GR)}

171 Generalized regression neural network (GR) is a memory neural network that is a variation to radial 172 basis function neural network (RBF) based on the statistical technique of kernel regression with a 173 dynamic network structure with powerful nonlinear mapping and robustness (Specht 1991). GR has 174 a high learning speed and is very useful for function approximation problems. For small sample 175 data, the prediction effect is excellent, and also unstable data can be processed. GR does not have $176 \mathrm{RBF}$ accuracy but has a major advantage in classification and fit, especially when data accuracy is 177 inappropriate. The GR model has a two-layer structure, the first layer being the hidden RBF layer 178 and the second layer being the linear output layer (Tayfur et al. 2020). For the hidden layer RBF, 179 the number of elements is equal to the number of training samples. The weight function of the 
180 hidden layer RBF is dist, which is used to determine the distance between the network input and

181 the weight value $\mathrm{IW}_{11}$ of this layer and is calculated from the following equation:

$$
\| \text { dist } \|_{j}=\sqrt{\sum_{i=1}^{R}\left(x_{i}-I W_{j i}\right)^{2} .} \quad(j=1.2 \ldots . M)
$$

182 In the hidden layer, network product function netprod multiplies the threshold $\mathrm{b}_{1}$ and $\|$ dist $\|$ output 183 to get net input $\mathrm{n}^{1}$. The net put $\mathrm{n}^{1}$ is passed to transfer function radbas. For the GR model, the 184 Gaussian function is used as the transfer function, namely,

$$
\begin{aligned}
\alpha_{j}^{1} & =\operatorname{radbas}\left(\text { netprod }\left(\| \text { dist } \|_{j} b_{1 . j}\right)\right) \\
& =\exp \left|-\frac{\left(n_{j}^{1}\right)^{2}}{2 \sigma_{j}^{2}}\right| \\
& =\exp \left|-\frac{\left(\| \text { dist } \|_{j} b_{1 . j}\right)^{2}}{2 \sigma_{j}^{2}}\right| \quad(j=1.2 \ldots . . M)
\end{aligned}
$$

185 In the above equation, $\sigma_{j}$ is a smoothing factor, also called the spread parameter, which calculates 186 the shape of RBF in the $j$ th hidden layer.

187 The normalized weight product function is used as the weight function in the linear output layer, 188 making the former layer's output with the weight value $\mathrm{IW}_{21}$ in this layer as the weight output. The 189 Purelin function is used as the transfer function for the output of the passed weight. The network 190 output is calculated from the following equation:

$$
y_{k}=\sum_{j=1}^{m} I W_{k j} \alpha_{j}^{1} \quad(k=1.2 \ldots . S)
$$

191 For the GR model, only the spread parameter should be specified. Due to the significant effect of 192 this parameter on the model's performance, the optimal value of this parameter must be determined. 


\subsection{Committee machine intelligent system (CMIS)}

194 To achieve the desired goals, different models are developed, and the best models are chosen as a 195 candidate, and other models are discarded. Under these circumstances, the cost incurred for the 196 discarded models is wasted. For this purpose, a committee machine can be built by combining 197 intelligent models and using the features of each of these models. This committee machine 198 intelligent system (CMIS) was introduced by Nilsson in 1965; it is a kind of artificial neural network 199 and uses division and conquers to solve problems.

200 In the committee machine method, the models are combined to provide a more accurate solution 201 (Hemmati-Sarapardeh et al. 2020). To combine the models, the linear combination method can be

202 used using simple averaging or weighted averaging (Hashem and Schmeiser 1970). Because all 203 models' contribution in the simple averaging method is the same, a satisfactory answer is not 204 obtained from this method because a more precise solution must contribute to the final model more. 205 For this purpose, the weighted average that combines models based on their precision, and the sum 206 of coefficients, the linear composition of the unit, can be used. In this research, added a bias term 207 to the equation and an improved weighted average is used. In the final model, any model's 208 contribution corresponds to that model's coefficient in the linear equation of the committee machine 209 intelligence system model.

\section{4. Optimization techniques}

\section{4.1 Levenberg-Marquardt algorithm (LM)}

212 The most common tool used to optimize the weight and bias of multilayer perceptron (MLP) and 213 cascade forward (CF) is the Levenberg-Marquardt (LM) algorithm, also known as the damped least214 squares method. This algorithm is used to solve nonlinear least-squares problems that find local 
215 minimums. This method does not require to calculate of the Hessian matrix and the gradient is

216 calculated from the following equation (Kisi and Uncuoğlu 2005):

$$
\begin{aligned}
& H=J^{T} J \\
& g=J^{T} e
\end{aligned}
$$

217 Where e stands a vector of network errors, and J expresses a Jacobian matrix. In the following 218 relation of updating the LM algorithm, the mentioned approximation with the Hessian matrix is 219 used:

$$
x_{k+1}=x_{k}-\left(J^{T} J-\eta I\right)^{-1} J^{T} e
$$

$220 \eta$ is a constant, and $x$ denotes connection weights. $\eta$ increases when an experimental step enlarges

221 the efficiency function.

\subsection{Bayesian Regularization algorithm (BR)}

223 The Bayesian Regularization (BR) training algorithm, according to Levenberg-Marquardt (LM)

224 optimization, updates weights and biases by minimizing a combination of squared errors and 225 weights (MacKay 1992). Afterward, BR calculates the right combination to develop a network with

226 superior generalization. Network weights are expressed as a training cost function by the BR 227 algorithm using the following equation:

$$
F(\omega)=\alpha E_{\omega}+\beta E_{D}
$$

228 In which $E_{D}$ and $E_{\omega}$ are the sum of the network errors and the sum of the squared network weights,

229 respectively, $F(\omega)$ denotes the objective function. In the BR optimizer, the network weights are 230 random variables in which the network weights and the training sets have a Gaussian distribution. $231 \alpha$ and $\beta$ Factors are objective function parameters that are clarified based on Bayes' theorem. 


\subsection{Scaled Conjugate Gradient algorithm (SCG)}

233 One of the basic features of the backpropagation algorithm is to reach the most negative gradient, 234 and it uses the adjustment of weights in the steepest descending direction., Along such a direction 235 a decrease in function performance is observed faster but does not cause faster convergence. In this 236 direction, a search is performed in the conjugate gradient (CG) method, which leads to faster 237 convergence than the steepest descending direction, and error minimization is maintained in 238 previous steps (Kisi and Uncuoğlu 2005).

$$
P_{0}=-g_{0}
$$

$239 \mathrm{P}$ is search direction, and $-\mathrm{g}_{0}$ denotes the steepest descent direction in the first iteration. This

240 direction is called conjugate direction, commonly used by conjugate gradient algorithms with the

241 search line. To evaluate the optimal distance to move in the current search direction, the step size

242 is determined by a line search technique (Kisi and Uncuoğlu 2005), which is shown by the following

243 equation:

$$
x_{k+1}=x_{k}+\alpha_{k} g_{k}
$$

244 In other words, the proper search direction is calculated in a way that conjugates with the previous 245 search direction.

$$
P_{k}=-g_{k}+\beta_{k} P_{k-1}
$$

246 Different versions of the conjugate algorithm are distinguished in the way that $\beta$ is calculated (Kisi 247 and Uncuoğlu 2005).

\section{$248 \quad 4.4$ Resilient Backpropagation algorithm (RB)}


249 The most widely used transfer functions in multilayer perceptron neural networks (MLP) are

250 Sigmoid and Tansig, which compress an infinite input range into a finite output. When using the

251 steepest descent to train the network using these activation functions, the slope is small when an

252 extensive input enters the function, leading to slight changes in weights and biases. The Resilient

253 backpropagation (RB) method is used to remove the adverse effect of the partial derivatives, which

254 is specified by the derivatives only for the direction of updating weights (Riedmiller and Braun

255 1993).

256 5. Results and discussion

\section{$257 \quad 5.1$ Models development}

258 In this research, 1542 data points of five piezometric wells have been used to model and predict the

259 groundwater level. The data used are divided into spatial (sediments and bedrock) and temporal

260 (March 21, 2019, to July 2, 2020). To consider the complexity of intelligent methods, 12 spatial

261 parameters (longitude, latitude, hydraulic conductivity of sediments, effective porosity of

262 sediments, the electrical resistivity of sediments, depth of sediments, surface level, hydraulic

263 conductivity of bedrock, effective porosity of bedrock, the electrical resistivity of bedrock, bedrock

264 level, and fault) and 6 time series parameters (day, month, year, drainage, evaporation, and rainfall)

265 have been used (Rajaee et al. 2019). 1377 data points have been used to develop four multilayer

266 perceptron neural network (MLP) models, four cascade forward neural network (CF) models, one

267 radial basis function neural network $(\mathrm{RBF})$ model, and one general regression neural network (GR)

268 model. MLP and CF models are each optimized by Levenberg-Marquardt (LM), Bayesian

269 Regularization (BR), Scaled Conjugate Gradient (SCG), and Resilient Backpropagation (RB)

270 methods. In all the mentioned models, $80 \%$ of the data were used for model training, and $20 \%$ of 
271 the data were used for model validity. The data are divided into testing and training sets using

272 random distributions to prevent the local accumulation of data.

273 The number of hidden layers, the type of transmission function, and the number of neurons in each

274 layer affects the efficiency of a developed model. Trial and error can be used to identify these

275 parameters. In this research, two hidden layers were used for multilayer perceptron neural network

276 (MLP) and cascade forward neural network (CF) methods, and one hidden layer was used for radial

277 basis function neural network $(\mathrm{RBF})$ and general regression neural network (GR) methods. Table

2784 shows the function used and the best architecture in terms of the number of neurons for each

279 model. Transfer functions are designed to correctly model the complex behavior of nonlinear input

280 and output datasets. The architecture for the MLP model consists of four numbers, the first and last

281 number being the number of inputs and outputs of the model, and the second and third numbers

282 being the number of neurons in the first and second hidden layers. The architecture for the CF model

283 consists of five numbers, the first and last number being the number of inputs and outputs of the

284 model, the third and fourth numbers being the number of hidden layer neurons in the first and

285 second. The second number is the number of neurons in the connections layer between input and 286 output. The architecture for the RBF model consists of 3 numbers, the first and last number being

287 the number of inputs and outputs of the model, and the second number being the number of 288 maximum neurons in the hidden layers. In Table 3, to compare the performance of the models, a 289 summary of statistical parameters including average absolute relative error (AARE, \%), average 290 relative error (ARE, \%), root mean square error (RMSE), standard deviation (SD), and model run 291 time for one iteration $(\mathrm{T}, \mathrm{Sec})$ for all developed models has been calculated. The mentioned 292 parameters are expressed as: 


$$
\begin{aligned}
& A A R E=\frac{100}{N} \sum_{i=1}^{N} \frac{\left|\mu_{r_{\text {exp.i }}}-\mu_{r_{\text {pred. } .} \mid}\right|}{\mu_{r_{\text {exp } . i}}} \\
& A R E=\frac{100}{N} \sum_{i=1}^{N} \frac{\mu_{r_{\text {exp.i }}}-\mu_{r_{\text {pred } . i}}}{\mu_{r_{\text {exp. } i}}} \\
& R M S E=\sqrt{\frac{1}{N} \sum_{i=1}^{N}\left(\mu_{r_{\text {exp } . i}}-\mu_{r_{\text {pred. } i}}\right)^{2}} \\
& S D=\sqrt{\frac{1}{N-1} \sum_{i=1}^{N}\left(\frac{\mu_{r_{\text {exp.i }}}-\mu_{r_{\text {pred. } i}}}{\mu_{r_{\text {exp } . i}}}\right)^{2}}
\end{aligned}
$$

293 Based on the information in Table 3, the multilayer perceptron neural network (MLP) and cascade

294 forward neural network (CF) created a model based on Levenberg-Marquardt (LM) and Bayesian 295 Regularization (BR) have more precise results than Resilient Backpropagation (RB) and Scaled 296 Conjugate Gradient (SCG) optimizers. The higher LM and BR optimizer accuracy possibly due to 297 using nonlinear least squares to find local minimums. Also, due to the inaccuracy of SCG and RB 298 methods compared to LM and BR methods, it is concluded that these optimizers are not suitable 299 for regression analysis. Since the efficiency of the created models is strongly influenced by the 300 initial biases and weights, the training of artificial neural networks with each optimizer using trial 301 and error was executed more than 50 times with dissimilar initial biases and weights, and the most 302 satisfactory results were chosen.

303 Radial basis function neural network (RBF) models consist of two key parameters: the number of 304 neurons and the spread coefficient. To determine these two parameters, trial and error have been 305 used. In this research, the number 5 for the coefficient of expansion and the number 30 for the 306 number of neurons have been used. As shown in Table 3, the RBF model is less accurate than other 
models but requires less run time the program, which can be used for models with many input data. To identify the optimal value for the coefficient of expansion and the number of neurons, the RBF model was implemented more than 100 times, and the best results were stored. The general regression neural network (GR) model, like the RBF model, has the spread coefficient parameter, which is 0.5 for this parameter by trial and error. Based on the information in Table 3, the developed GR model has a value of $0.035 \%$ average absolute relative error (AARE, \%). This model like RBF models requires less time to run than other methods. RBF and GR methods are faster than the multilayer perceptron neural network (MLP) and cascade forward neural network (CF) methods due to their monolayer. The results of Table 3 have been used to rank the proposed models based on having the highest accuracy:

$$
\begin{gathered}
\mathrm{MLP}-\mathrm{LM}>\mathrm{CF}-\mathrm{BR}>\mathrm{MLP}-\mathrm{BR}>\mathrm{MLP}-\mathrm{RB}>\mathrm{CF}-\mathrm{LM}>\mathrm{CF}-\mathrm{RB}>\mathrm{CF}-\mathrm{SCG} \\
>\mathrm{MLP}-\mathrm{SCG}>\mathrm{GR}>\mathrm{RBF}
\end{gathered}
$$

After developing different models using 1377 data points in the previous step, 165 extra validation data points have been used to identify the best models and their combination and to develop the proposed committee machine intelligence system (CMIS) model. The statistical results of the developed models using 165 extra validation data points are shown in Table 4. The results show high accuracy of radial basis function neural network (RBF), multilayer perceptron neural network using Levenberg-Marquardt optimizer (MLP-LM), and cascade forward neural network using Bayesian Regularization optimizer (CF-BR) compared to other models. The three models developed with the lowest average absolute relative error (AARE, \%) value are combined with a CMIS. To detect the optimal coefficients of this model, multiple linear regression is used, which is shown in the next equation: 


$$
\mu_{\mathrm{CMIS}}=\alpha_{1} \mu_{\mathrm{MLP}-\mathrm{LM}}+\alpha_{2} \mu_{\mathrm{CF}-\mathrm{BR}}+\alpha_{3} \mu_{\mathrm{RBF}}+\alpha_{4}
$$

329 In the above equation, $\alpha_{1}$ to $\alpha_{4}$ are as follows:

$$
\alpha_{1}=0.525543 ; \alpha_{2}=0.522345 ; \alpha_{3}=-0.04743 ; \alpha_{4}=-0.7539 ;
$$

331 CMIS model proposed in this research showed in Fig. 3.

\section{$332 \quad 5.2$ Evaluation of the validity and precision of the developed models}

333 Statistical parameters such as average absolute relative error (AARE, \%), average relative error 334 (ARE, \%), root mean square error (RMSE), standard deviation (SD) for developed models are 335 shown in Table 4. Some models, such as radial basis function neural network (RBF), which has the 336 highest AARE value in Table 3, but here with one of the lowest error values, is one of the best

337 models to predict, indicating accurate learning between input and output relationships. Other 338 models, such as the cascade forward neural network using Scaled Conjugate Gradient optimizer 339 (CF-SCG), which do not have a good prediction of groundwater level from extra validation data, 340 probably due to overtraining and memorizing input-output rules or maybe stuck in local optimizations. AARE value of about $0.11 \%$ indicates that the proposed committee machine

342 intelligence system (CMIS) model is the most precise model for groundwater level forecasting, 343 among other developed models. In addition to the CMIS model, RBF, cascade forward neural 344 network using Bayesian Regularization optimizer (CF-BR), and multilayer perceptron neural 345 network using Levenberg-Marquardt optimizer (MLP-LM) models alone have an acceptable ability 346 to predict groundwater levels. To visually confirm the precision of the developed CMIS model 347 using 1377 data points, the cross plot of the experimental data versus predicted relative groundwater 348 level are plotted in Fig. 4. and the relative error distribution curve for testing and training dataset is 349 drawn in Fig. 5. The high concentration of test and train data around the unit slope indicates the 
350 high accuracy of the CMIS model prediction. In Fig. 5, the maximum relative error of the predicted 351 and experimental data values is $0.15 \%$. Most of the dataset points are located about the zero-error 352 line for the tentative relative groundwater level. This figure confirms the stability between the 353 experimental data and the CMIS model prediction. Fig. 6. shows the actual groundwater level and 354 the prediction of groundwater level for 165 days ahead by the developed models with the least 355 AARE value.

356 The lowest average absolute relative error (AARE, \%), average relative error (ARE, \%), root mean 357 square error (RMSE), standard deviation (SD) values indicate an acceptable estimate by the 358 developed committee machine system intelligence (CMIS) model compared to other developed 359 models. A detailed error analysis has been used to more compare the efficiency of the proposed 360 CMIS model and other developed models for groundwater level prediction. The AARE (\%) in 361 Fig.7-11 is drawn as a function of the input parameters that have the most significant impact on the 362 groundwater surface. According to Table 1, the highest relevancy is related to the electrical 363 resistivity of sediments, depth of sediments, the electrical resistivity of bedrock, hydraulic 364 conductivity of bedrock, and effective porosity of sediments. For this purpose, error analysis was 365 performed by dividing the entire data into four groups to indicate the models' precision in various 366 ranges of crucial parameters.

367 Fig. 7. compares the average absolute relative error (AARE, \%) of dissimilar models in four ranges 368 of electrical resistivity of sediments. The maximum AARE value of $0.021 \%$ for the proposed 369 committee machine intelligence system (CMIS) model indicates its very high capability to predict 370 the groundwater level in the whole range of electrical resistivity of sediments. As shown from this 371 figure, developed models for the range of 21 to 32 (ohm-m) have a lower AARE error than other 372 values. Fig. 8. shows the AARE (\%) of the developed models in four ranges of the depth of 
373 sediments. This figure clearly shows a lower error of the CMIS model than all models. Close to the

374 surface in the range of 130 to 150 meters, the AARE value is the lowest. Fig. 9. presents the

375 efficiency of the developed models over dissimilar ranges of the electrical resistivity of bedrock.

376 As can be easily seen, as in Figure 7, the lowest AARE value here is in the range of 21 to 30 (ohm-

$377 \mathrm{~m})$. Besides, the CMIS model has the lowest AARE in all four ranges. Fig. 10. depicts the AARE

378 of the developed models for dissimilar ranges of hydraulic conductivity of bedrock. Again, the

379 proposed CMIS is accurate in the entire range, especially in the range of 18 to 19 (m/day). The

380 effect of porosity of sediments in Fig. 11. in four ranges shows a low AARE value for all models,

381 mainly CMIS. In all ranges, the AARE values are close to each other models and below $0.05 \%$.

382 To better compare the models, the cumulative frequency of average absolute relative error for the 383 developed committee machine intelligence system (CMIS) model and all other models is shown in 384 Fig. 12. According to this figure, about $85.5 \%$ of the groundwater level predicted by the developed 385 CMIS model has an average absolute relative error (AARE, \%) of less than $0.03 \%$. Other developed 386 multilayer perceptron neural network using Levenberg-Marquardt optimizer (MLP-LM), 387 multilayer perceptron neural network using Bayesian Regularization optimizer (MLP-BR), and 388 cascade forward neural network using Bayesian Regularization optimizer (CF-BR) models have $38982.4 \%, 83.1 \%$, and $82.9 \%$ errors. The results of this figure further indicate the success of the 390 proposed CMIS method compared to other developed methods for groundwater level prediction.

\section{$391 \quad 5.3$ Trend analysis of the developed model}

392 Trend analysis in a hydrogeological time series can be a practical tool to study groundwater level 393 fluctuations (Halder et al. 2020). By examining the groundwater level predicted in Figure 6, 394 significant changes are seen on days 40 to 110 , indicating the pumping well in the southeastern part 
395 of the mine. For this reason, considering that the drainage time series is a parameter affecting the

396 groundwater level, the physically expected trends of the groundwater level by changing the

397 drainage is investigated by the models developed in Fig 13. As can be seen in this figure, the relative

398 groundwater level decreases with the drainage increases. All developed models can record the 399 expected trend with drainage changes. This figure also shows the accuracy of the proposed 400 committee machine intelligence system (CMIS) model compared to other methods.

\subsection{Sensitivity analysis on models' inputs}

402 Sensitivity analysis is important from the perspective that uncertainty in model inputs affects

403

404

405

406 407 408

409 following equation:

$$
r\left(\operatorname{Inp}_{k} \cdot \mu\right)=\frac{\left|\sum_{i=1}^{n}\left(\operatorname{Inp}_{k . i}-\operatorname{Inp}_{\text {ave } . k}\right)\left(\mu_{i}-\mu_{\text {ave }}\right)\right|}{\sqrt{\sum_{i=1}^{n}\left(\operatorname{Inp}_{k . i}-\operatorname{Inp}_{\text {ave.k }}\right)^{2} \sum_{i=1}^{n}\left(\mu_{i}-\mu_{\text {ave }}\right)^{2}}}
$$

410 Where $\operatorname{Inp}_{\mathrm{k} . \mathrm{i}}$ shows the $i$ th value, and Inp $\mathrm{Iave.k}_{\text {is }}$ the average value of $k$ th input, respectively ( $\mathrm{k}$ 411 represents the model inputs). $\mu_{\mathrm{i}}$ and $\mu_{\text {ave }}$ show the $i$ th value and the average value of the predicted 412 output (Hemmati-Sarapardeh et al. 2020). The Fig. 14. shows the value of the relevancy factor. The 413 obtained numbers show that spatial parameters have a more significant effect on groundwater 414 surface than temporal parameters. The most influential spatial parameters on the groundwater level 
415 are the electrical resistivity of sediments, depth of sediments, the electrical resistivity of bedrock,

416 hydraulic conductivity of the bedrock, and effective porosity of sediments. Among the temporal

417 parameters, drainage volume has the most significant impact on groundwater level due to pumping

418 wells in the pit mine's southeastern region.

\subsection{Outlier diagnosis and applicability domain of the model}

420 Outliers commonly appear in a comprehensive set of experimental data and differ from the bulk of

421 the data. Because such a dataset can affect the reliability and precision of experimental models, thus

422 finding this type of dataset is essential in developing models. One of the practical advantages of

423 this analysis is that by carefully examining the outlier data, a good view of the model constraint can

424 be provided, which may be due to the ignoring of some justifiable effects. In this research, the

425 leverage approach has been used to determine and eliminate outliers, including calculating the

426 model deviation from the relevant experimental data. The computed model deviations are placed in

427 a Hat matrix and are called "standardized cross-validated residuals" (Rousseeuw and Leroy 1987).

428 William's plot is plotted in Fig. 15. for the resulting by the developed committee machine 429 intelligence system (CMIS) model for 1542 groundwater level predictions data. Due to the location

430 of most of the predicted points in the feasibility domain of the proposed model $(0 \leq$ hat $\leq 0.0414$

431 and $-3 \leq \mathrm{R} \leq 3$ ), statistical validity and high reliability of the developed CMIS model are shown.

432 About $2.65 \%$ of the points are outside the acceptable range of the model, which can be ignored due

433 to the number of data points used in the model's development. Points in the range of $\mathrm{R}<-3$ or

$434 \mathrm{R}>3$ are defined as "Bad High Leverage" regardless of their hat value compared to hat*

435 (Hemmati-Sarapardeh et al. 2016). These data may be well predicted, but due to the difference with

436 a large amount of data, but, are outside the acceptable range of the model. 


\section{Conclusions}

438 In this research, data from five piezometric wells includes 1542 data points around Gohar Zamin

439 Iron Ore Mine, located in Sirjan, Iran, have been used to predict the groundwater level. 1377 spatial

440 (sediment and bedrock) and temporal data points (276 days) have been used to develop ten

441 supervised learning models. 165 extra validation data points have been used to identify the best

442 models with less average absolute relative error (AARE, \%) value, combine them into a single

443 model, and develop the proposed committee machine intelligence system (CMIS) model. The

444 results of this research can be concluded:

445 1. Almost all developed models with 1377 data points can predict groundwater levels with 446 acceptable AARE values in the range of 0.017 to $0.043 \%$. The lowest error is related to multilayer 447 perceptron neural network using Levenberg-Marquardt optimizer (MLP-LM), cascade forward 448 neural network using Bayesian Regularization optimizer (CF-BR), and multilayer perceptron neural 449 network using Bayesian Regularization (MLP-BR) models, all of which have average absolute 450 relative error (AARE, \%) values below $0.018 \%$.

451 2. The running time of different algorithms with similar conditions shows that general regression

452 neural network (GR) and radial basis function neural network (RBF) algorithms are significantly 453 faster due to these models' monolayer.

454 3. Developed models using 1377 data points to predict groundwater levels for 165 extra validation 455 data points showed almost similar results, except that the RBF model has the lowest average 456 absolute relative error (AARE, \%). A lower AARE value for the radial basis function neural 457 network (RBF) model indicates acceptable learning of the input-output relationships. Developed 458 models predict groundwater levels for these 165 extra validation data points with AARE values 
459 between 0.12 and $0.84 \%$. A combination of three models, RBF, cascade forward neural network 460 using Bayesian Regularization optimizer (CF-BR), and multilayer perceptron neural network using 461 Levenberg-Marquardt optimizer (MLP-LM), is used to develop the committee machine intelligence 462 system (CMIS) model.

463 4. The developed committee machine intelligence system (CMIS) model has a better performance 464 than all other models and can predict the groundwater level with an average absolute relative error 465 (AARE, \%) value of about $0.11 \%$.

466 5. About $85.5 \%$ of the prediction of groundwater level has an average absolute relative error 467 (AARE, \%) value below $0.03 \%$, which indicates the statistical validity of this method. Data that are 468 out of the applicability domain of the proposed committee machine intelligence system (CMIS) are 469 about $2.65 \%$, which indicates the high accuracy of this method.

470 6. The electrical resistivity of sediments, Depth of sediments, the electrical resistivity of bedrock,

471 hydraulic conductivity of bedrock, and effective porosity of sediments of the 18 input parameters 472 are the most influential input parameters on the groundwater level, respectively. Also, trend analysis 473 for drainage parameters shows a decrease in groundwater level with increasing drainage due to 474 pumping wells' activity in the southeastern part of the mine.

475

\section{Declaration}

\section{Ethical Approval}

478 All work is compliance with Ethical Standards.

\section{Consent to Participate}


480 Authors give their permission.

\section{Consent to Publish}

482 Authors give their permission.

483 Authors Contributions

484 All authors have contributed to the paper. Amirhossein Najafabadipour has written the paper and

485 Gholamreza Kamali and Hossein Nezamabadi-pour reviewed and improved the manuscript several 486 times.

487 Funding

488 Not applicable

489 Competing Interests

490 Not applicable

491 Availability of data and materials

492 Not applicable 


\section{References}

Brawner CO (1986) Groundwater and coal mining Mining Science and Technology 3:187-198 doi:https://doi.org/10.1016/S0167-9031(86)90331-2

Broomhead D, Lowe D (1988) Radial basis functions, multi-variable functional interpolation and adaptive networks ROYAL SIGNALS AND RADAR ESTABLISHMENT MALVERN (UNITED KINGDOM) RSRE-MEMO-4148

Cybenko G (1989) Approximation by superpositions of a sigmoidal function Mathematics of Control, Signals and Systems 2:303-314 doi:10.1007/BF02551274

Fahlman S, Lebiere C (1989) The cascade-correlation learning architecture Advances in neural information processing systems 2:524-532

Golestani Kermani S, Sayari S, Kisi O, Zounemat-Kermani M (2019) Comparing data driven models versus numerical models in simulation of waterfront advance in furrow irrigation Irrigation Science 37:547-560 doi:10.1007/s00271-019-00635-5

Halder S, Roy MB, Roy PK (2020) Analysis of groundwater level trend and groundwater drought using Standard Groundwater Level Index: a case study of an eastern river basin of West Bengal, India SN Applied Sciences 2:507 doi:10.1007/s42452-020-2302-6

Hashem S, Schmeiser B (1970) Approximating a Function and its Derivatives Using MSE-Optimal Linear Combinations of Trained Feedforward Neural Networks 1

Hayder G, Solihin MI, Mustafa HM (2020) Modelling of River Flow Using Particle Swarm Optimized Cascade-Forward Neural Networks: A Case Study of Kelantan River in Malaysia Applied Sciences $10: 8670$

Hebb DO (1949) The organization of behavior: a neuropsychological theory. J. Wiley; Chapman \& Hall, Hemmati-Sarapardeh A, Ameli F, Dabir B, Ahmadi M, Mohammadi AH (2016) On the evaluation of asphaltene precipitation titration data: Modeling and data assessment Fluid Phase Equilibria 415:88-100 
Hemmati-Sarapardeh A, Larestani A, Nait Amar M, Hajirezaie S (2020) Chapter 1 - Introduction. In: Hemmati-Sarapardeh A, Larestani A, Nait Amar M, Hajirezaie S (eds) Applications of Artificial Intelligence Techniques in the Petroleum Industry. Gulf Professional Publishing, pp 1-22. doi:https://doi.org/10.1016/B978-0-12-818680-0.00001-1

Hemmati-Sarapardeh A, Varamesh A, Husein MM, Karan K (2018) On the evaluation of the viscosity of nanofluid systems: Modeling and data assessment Renewable and Sustainable Energy Reviews 81:313-329 doi:https://doi.org/10.1016/j.rser.2017.07.049

Hong $\mathrm{H}$ et al. (2020) Radial basis function artificial neural network (RBF ANN) as well as the hybrid method of RBF ANN and grey relational analysis able to well predict trihalomethanes levels in tap water Journal of Hydrology 591:125574 doi:https://doi.org/10.1016/j.jhydrol.2020.125574

Kisi O, Uncuoğlu E (2005) Comparison of three back-propagation training algorithms for two case studies Indian Journal of Engineering and Materials Sciences 12

Kouziokas GN, Chatzigeorgiou A, Perakis K (2018) Multilayer Feed Forward Models in Groundwater Level Forecasting Using Meteorological Data in Public Management Water Resources Management 32:5041-5052 doi:10.1007/s11269-018-2126-y

MacKay DJ (1992) Bayesian interpolation Neural computation 4:415-447

Malekpour MM, Mohammad Rezapour Tabari M (2020) Implementation of supervised intelligence committee machine method for monthly water level prediction Arabian Journal of Geosciences 13:1049 doi:10.1007/s12517-020-06034-X

Menad NA, Noureddine Z, Hemmati-Sarapardeh A, Shamshirband S (2019) Modeling temperature-based oil-water relative permeability by integrating advanced intelligent models with grey wolf optimization: Application to thermal enhanced oil recovery processes Fuel 242:649-663 doi:https://doi.org/10.1016/j.fuel.2019.01.047

Meshram SG, Singh VP, Kisi O, Karimi V, Meshram C (2020) Application of Artificial Neural Networks, Support Vector Machine and Multiple Model-ANN to Sediment Yield Prediction Water Resources Management 34:4561-4575 doi:10.1007/s11269-020-02672-8 
Nilsson N (1965) Learning machines

Pal J, Chakrabarty D (2020) Assessment of artificial neural network models based on the simulation of groundwater contaminant transport Hydrogeology Journal 28:2039-2055 doi:10.1007/s10040-02002180-4

Parisouj P, Mohebzadeh H, Lee T (2020) Employing Machine Learning Algorithms for Streamflow Prediction: A Case Study of Four River Basins with Different Climatic Zones in the United States Water Resources Management 34:4113-4131 doi:10.1007/s11269-020-02659-5

Rajaee T, Ebrahimi H, Nourani V (2019) A review of the artificial intelligence methods in groundwater level modeling Journal of Hydrology $572: 336-351$ doi:https://doi.org/10.1016/j.jhydrol.2018.12.037

Riedmiller MA, Braun H (1993) A direct adaptive method for faster backpropagation learning: the RPROP algorithm IEEE International Conference on Neural Networks:586-591 vol.581

Rousseeuw PJ, Leroy AM (1987) Robust regression and outlier detection. John Wiley \&amp; Sons, Inc., Santos Finck J, Correa Pedrollo O (2021) Facing Losses of Telemetric Signal in Real Time Forecasting of Water Level using Artificial Neural Networks Water Resources Management doi:10.1007/s11269021-02782-X

Singh RN, Atkins AS (1985) Analytical techniques for the estimation of mine water inflow International Journal of Mining Engineering 3:65-77 doi:10.1007/BF00881342

Specht DF A general regression neural network

Tayfur G, Aksoy H, Eris E (2020) Prediction of rainfall runoff-induced sediment load from bare land surfaces by generalized regression neural network and empirical model Water and Environment Journal 34:66-76 doi:https://doi.org/10.1111/wej.12442

Xu X, Huang G, Qu Z, Pereira LS (2011) Using MODFLOW and GIS to Assess Changes in Groundwater Dynamics in Response to Water Saving Measures in Irrigation Districts of the Upper Yellow River Basin Water Resources Management 25:2035-2059 doi:10.1007/s11269-011-9793-2 


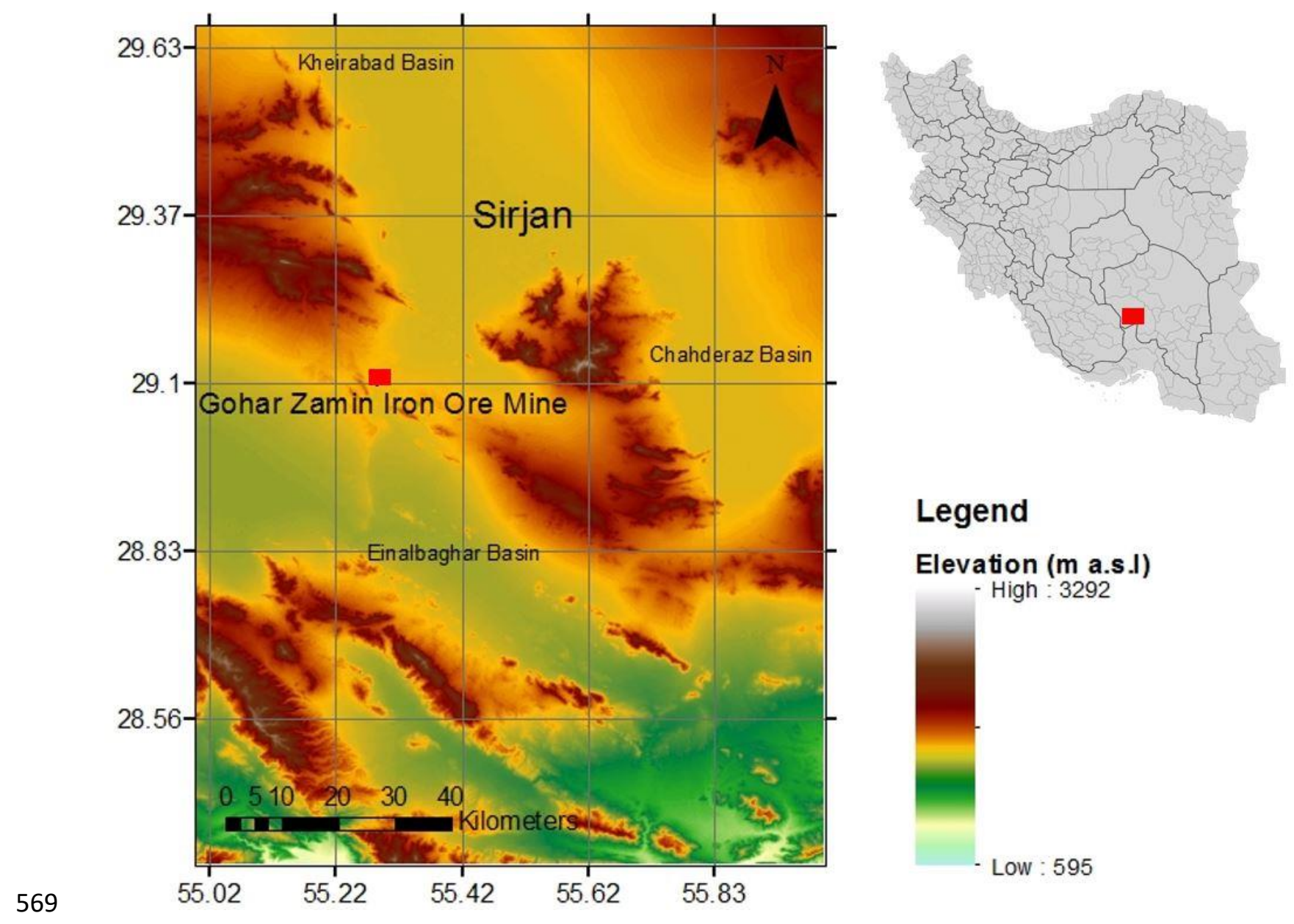

Fig. 1. Study area and location of Gohar-Zamin Iron Ore Mine.

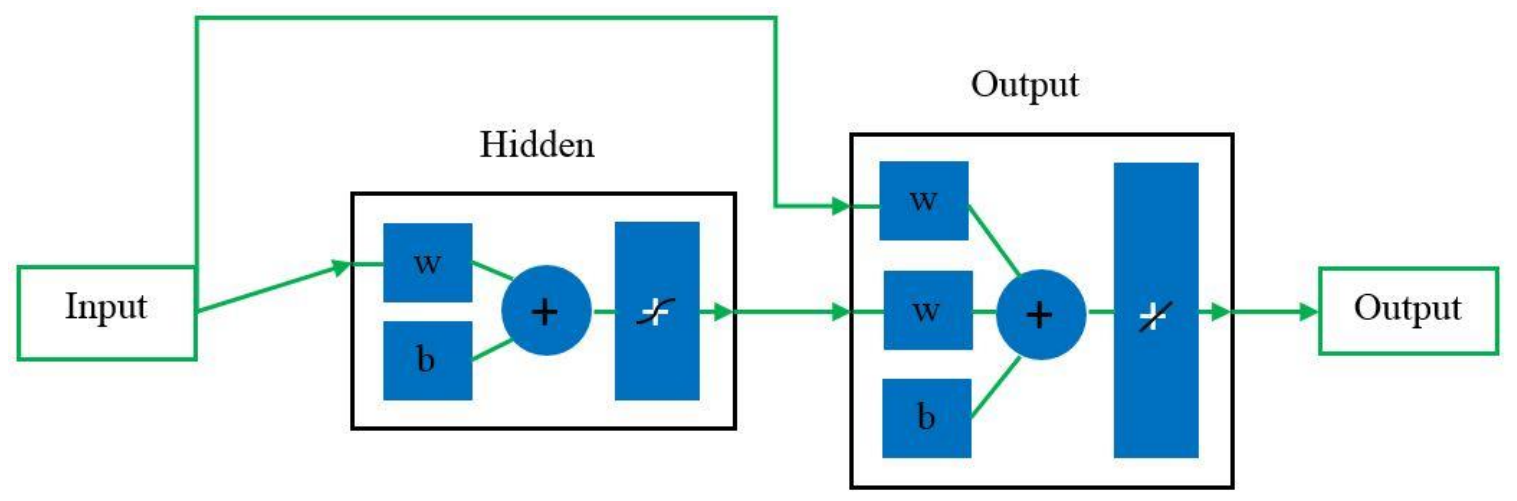

Fig. 2. The architecture of CF neural network. 


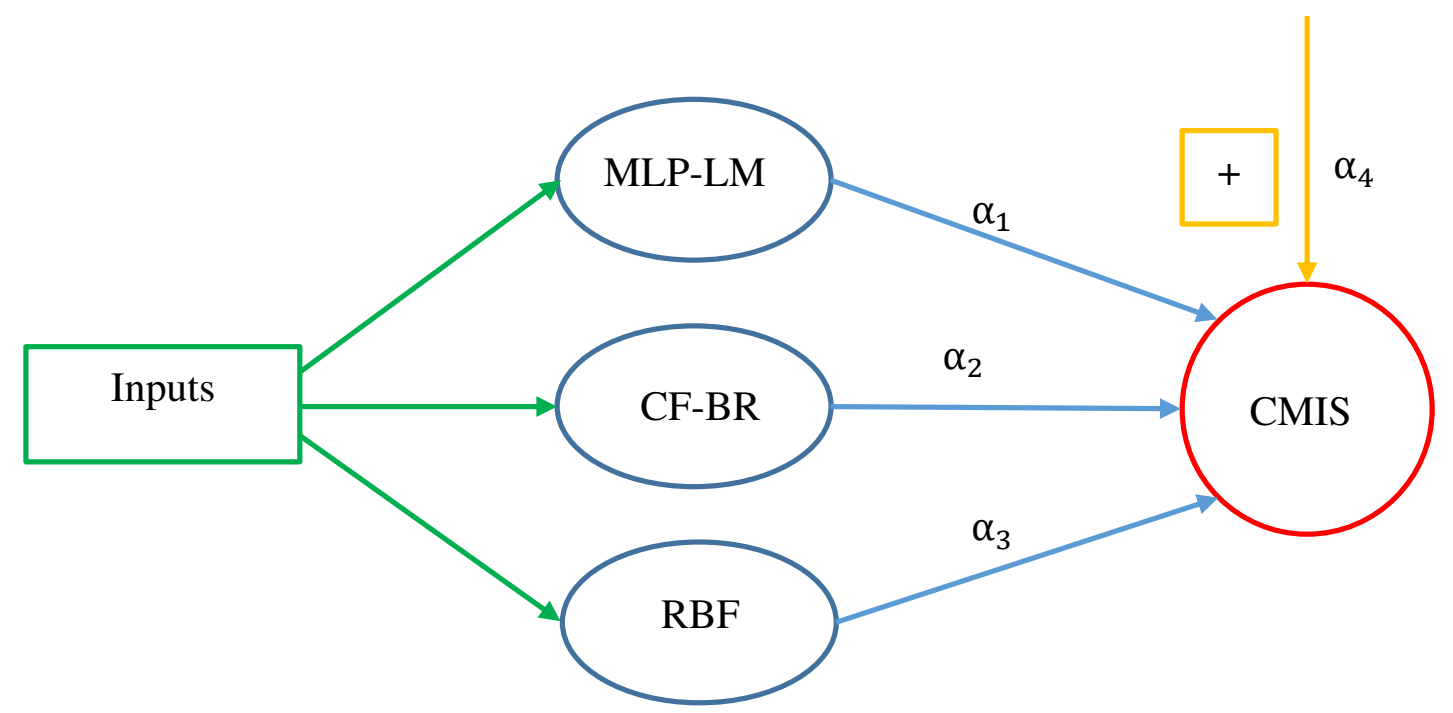

Fig. 3. The architecture of the proposed CMIS model.

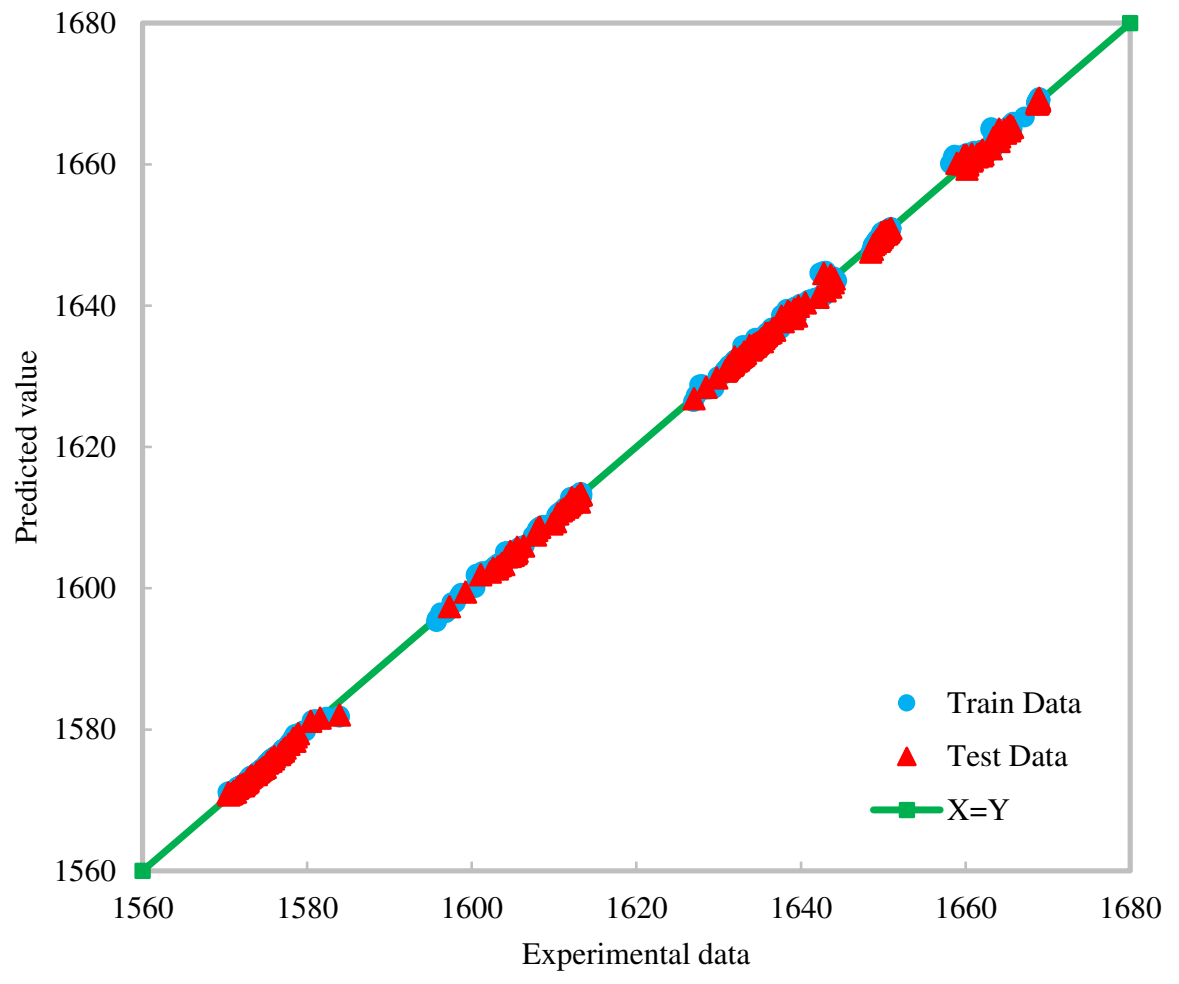

584 Fig. 4. Cross plot of predicted relative groundwater level versus tentative relative groundwater level for test and train data using 1377 data points. 


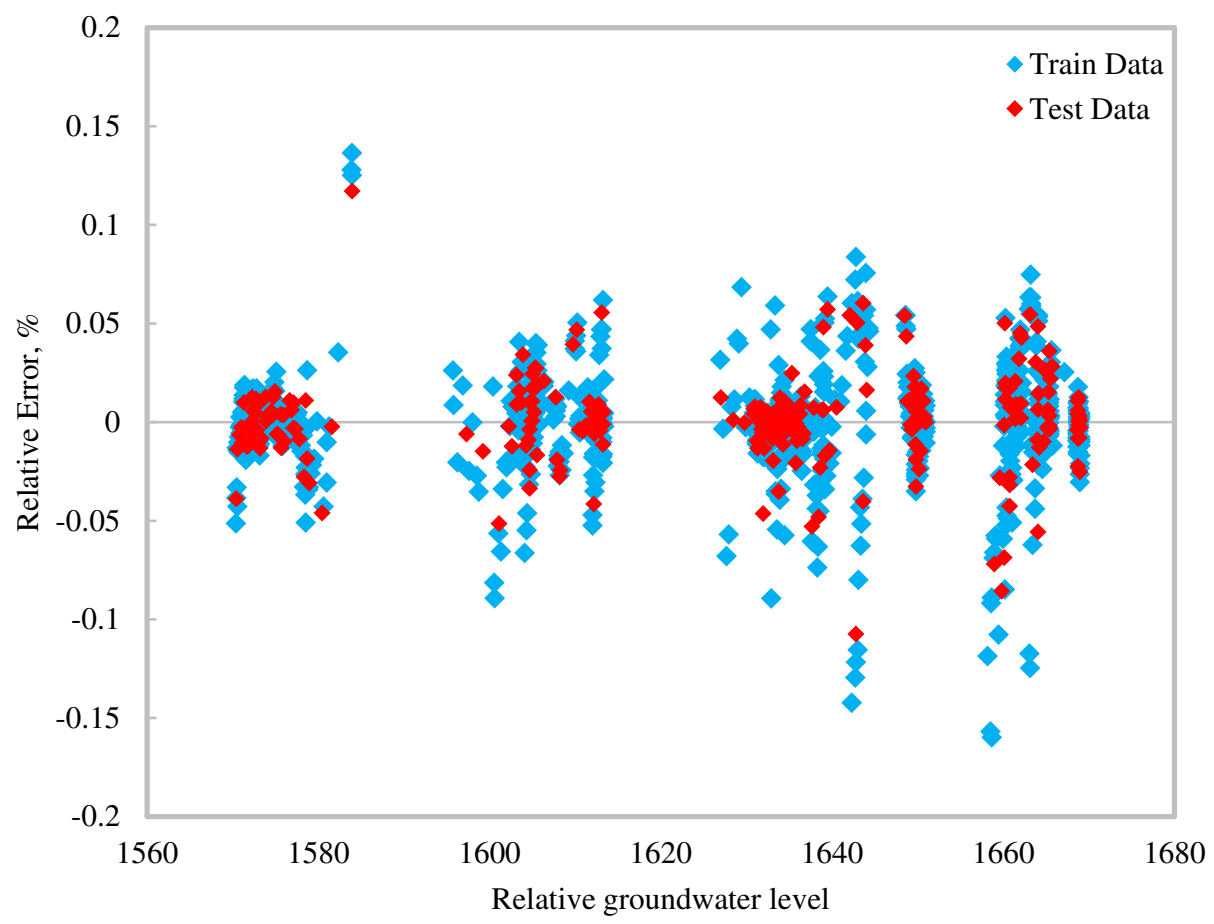

587 Fig. 5. The relative error between the tentative and predicted relative groundwater level versus 588 tentative relative groundwater level for train and test data using 1377 data points.

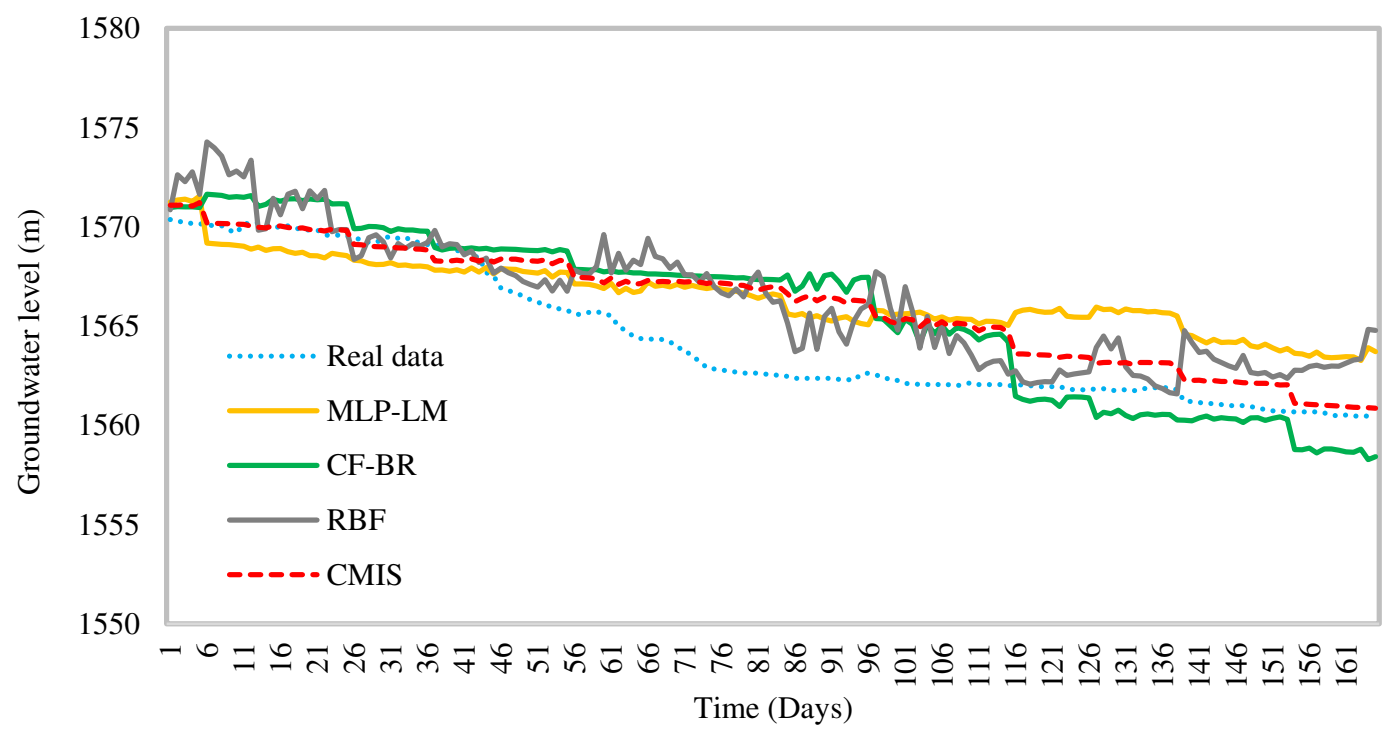

Fig. 6. Prediction of groundwater level for different models using 165 extra data points. 


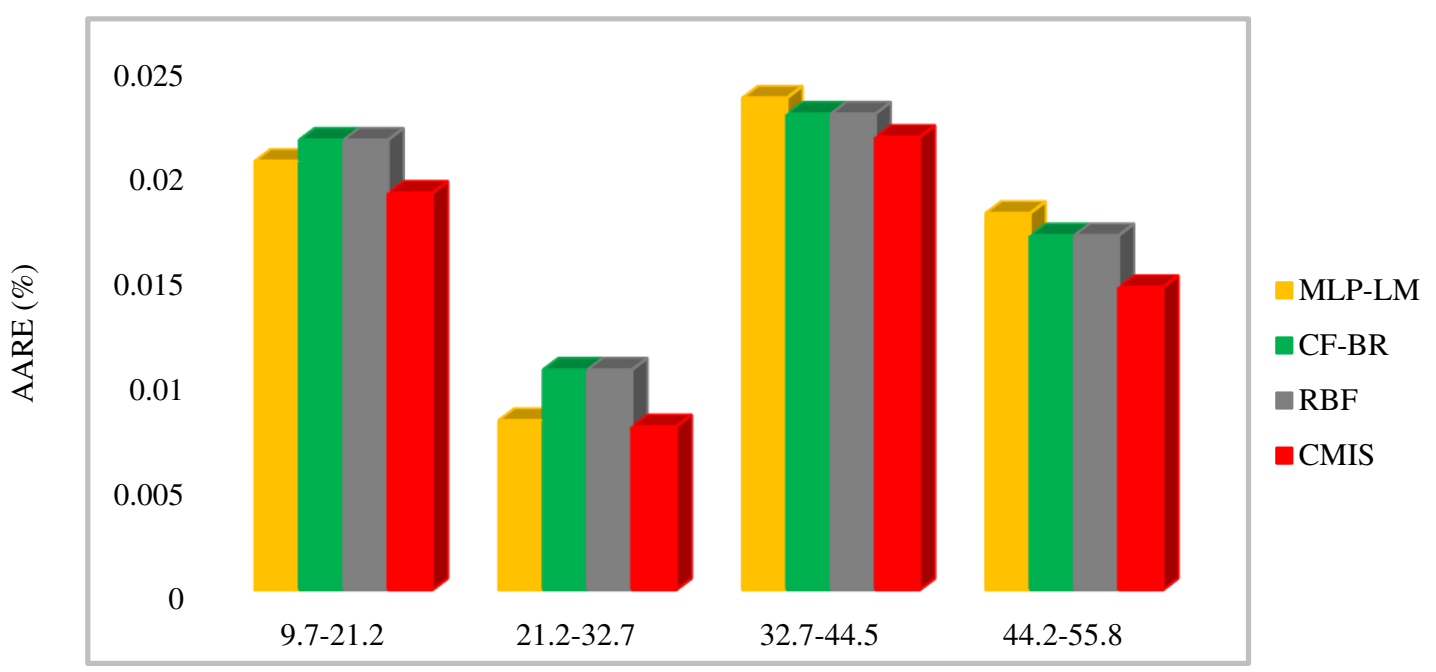

Electrical resistivity of sediments (ohm-m)

Fig. 7. AARE (\%) for the developed CMIS model and best models for different electrical resistivity of sediments ranges.

595

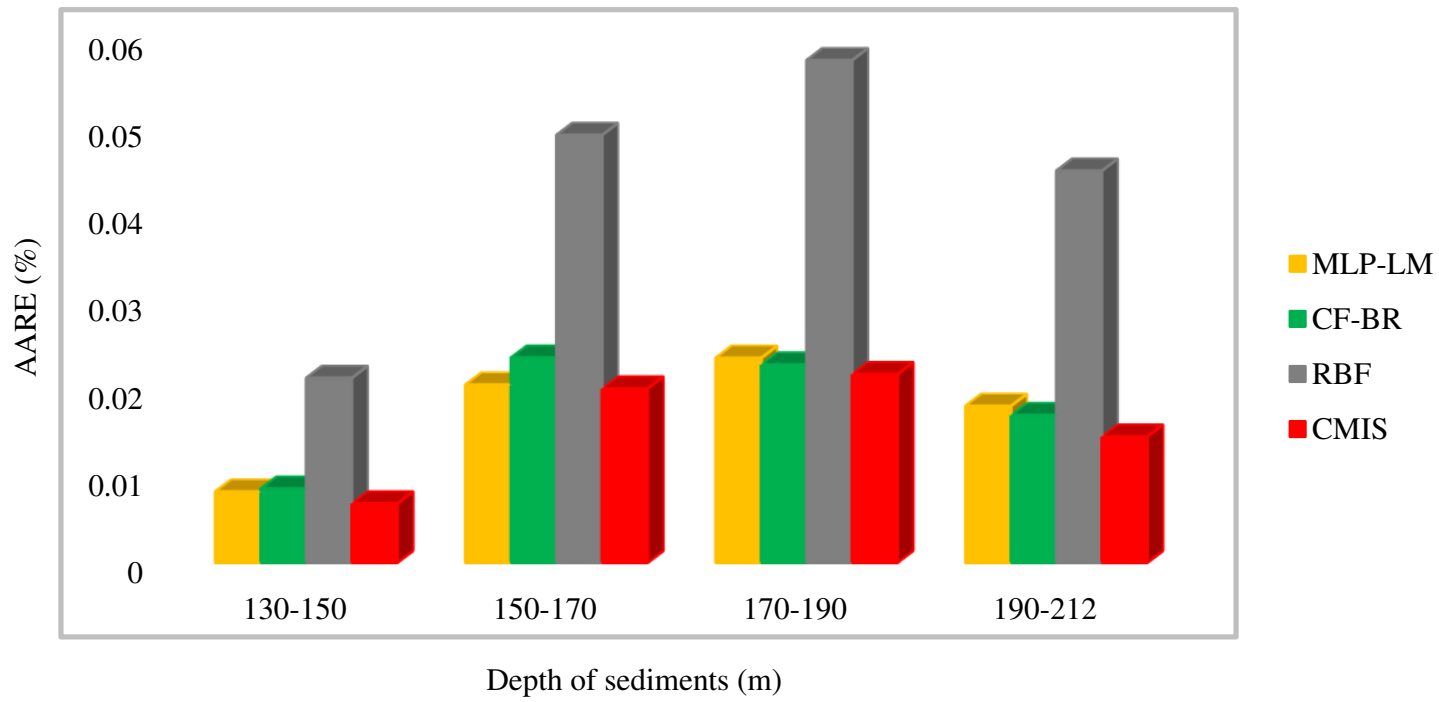

597 Fig. 8. AARE (\%) for the developed CMIS model and best models for the depth of sediments ranges. 


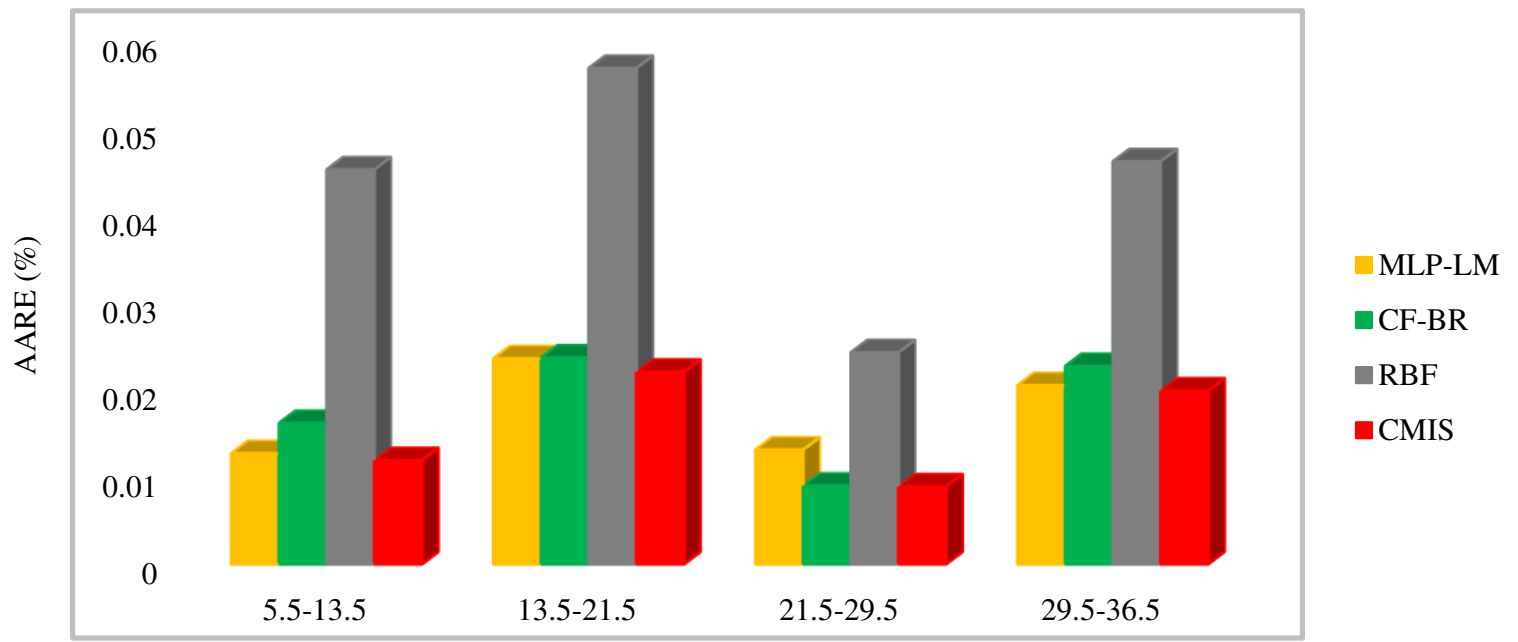

Electrical resistivity of bedrock (ohm-m)

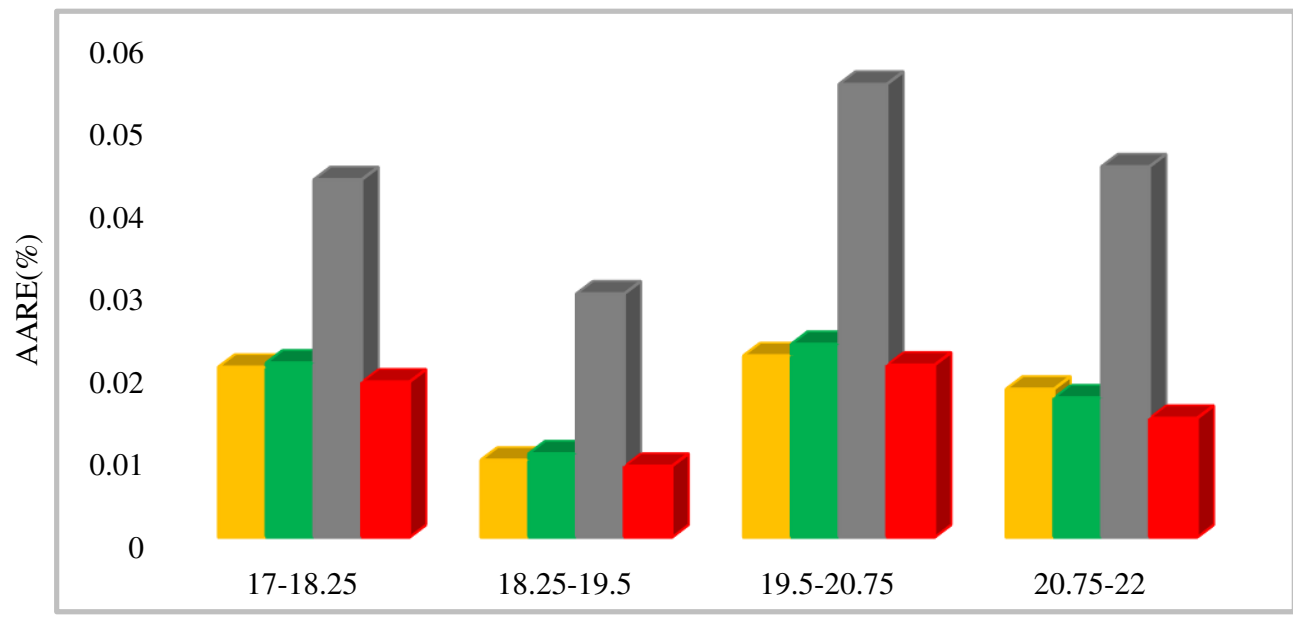

MLP-LM

- CF-BR

$\mathrm{RBF}$

- CMIS

Hydraulic conductivity of bedrock (m/day)

605 Fig. 10. AARE (\%) for the developed CMIS model and best models for hydraulic conductivity of bedrock ranges. 

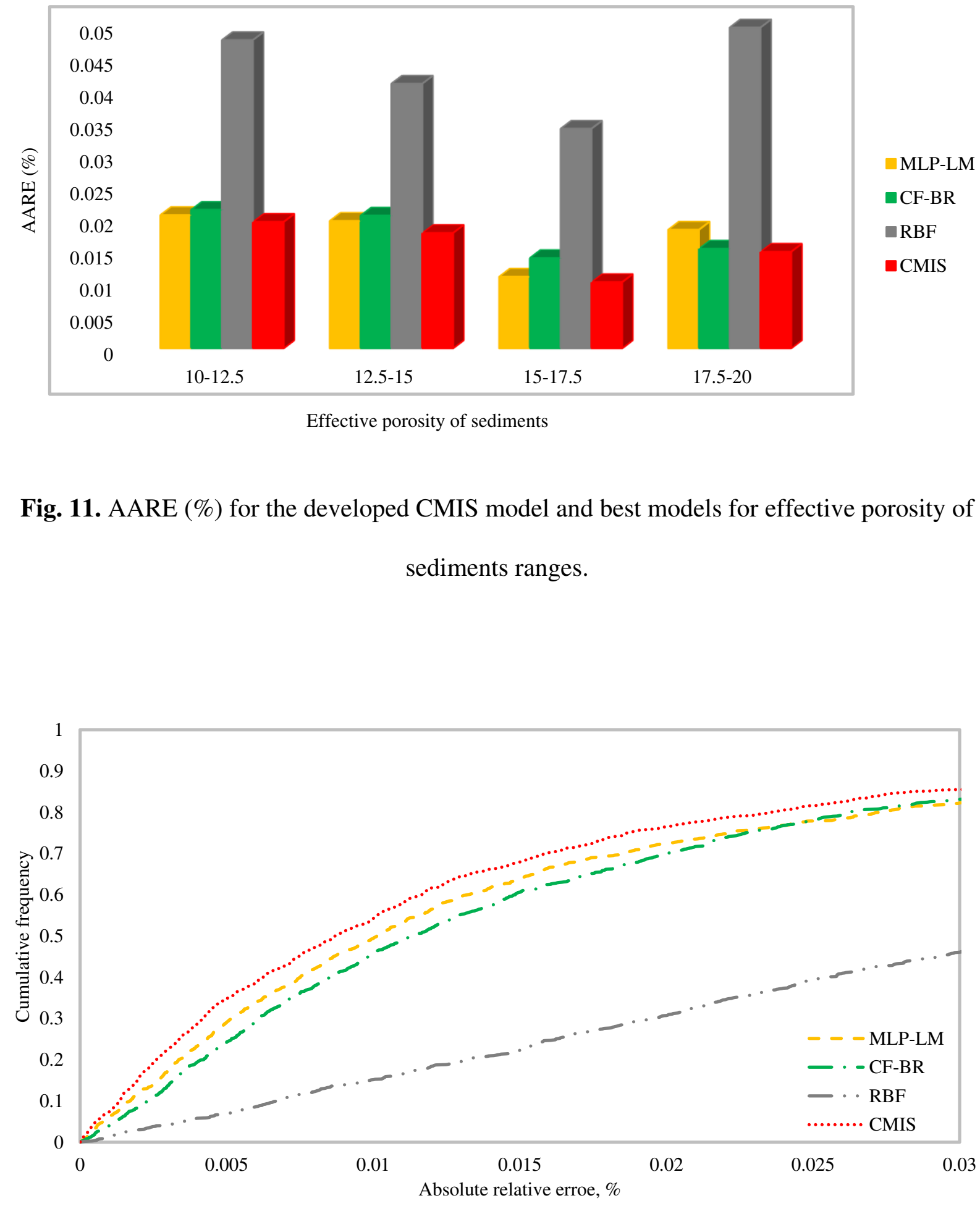

Fig. 12. Cumulative frequency versus absolute relative error for all developed models. 


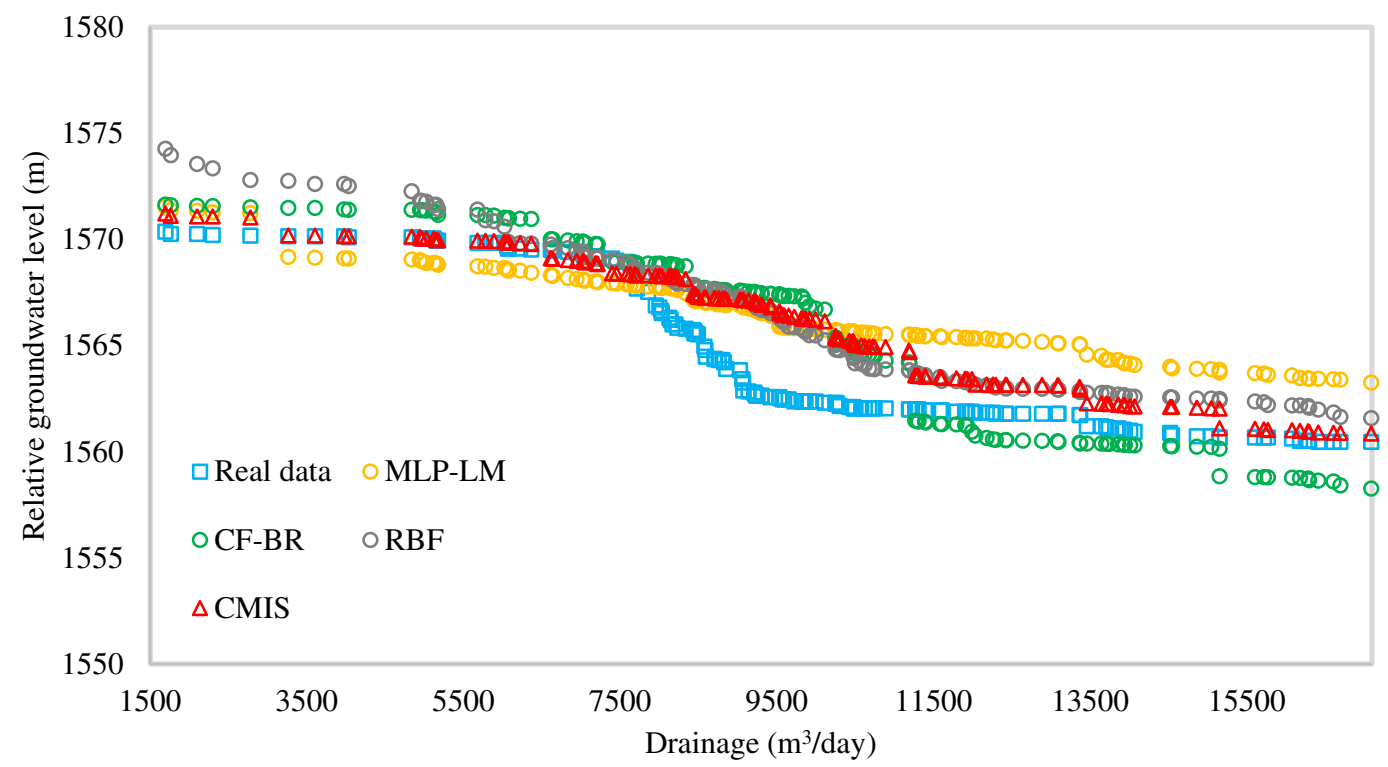

616 Fig. 13. Comparing the relative variation with drainage for the proposed CMIS model and other

617 developed models with real data.

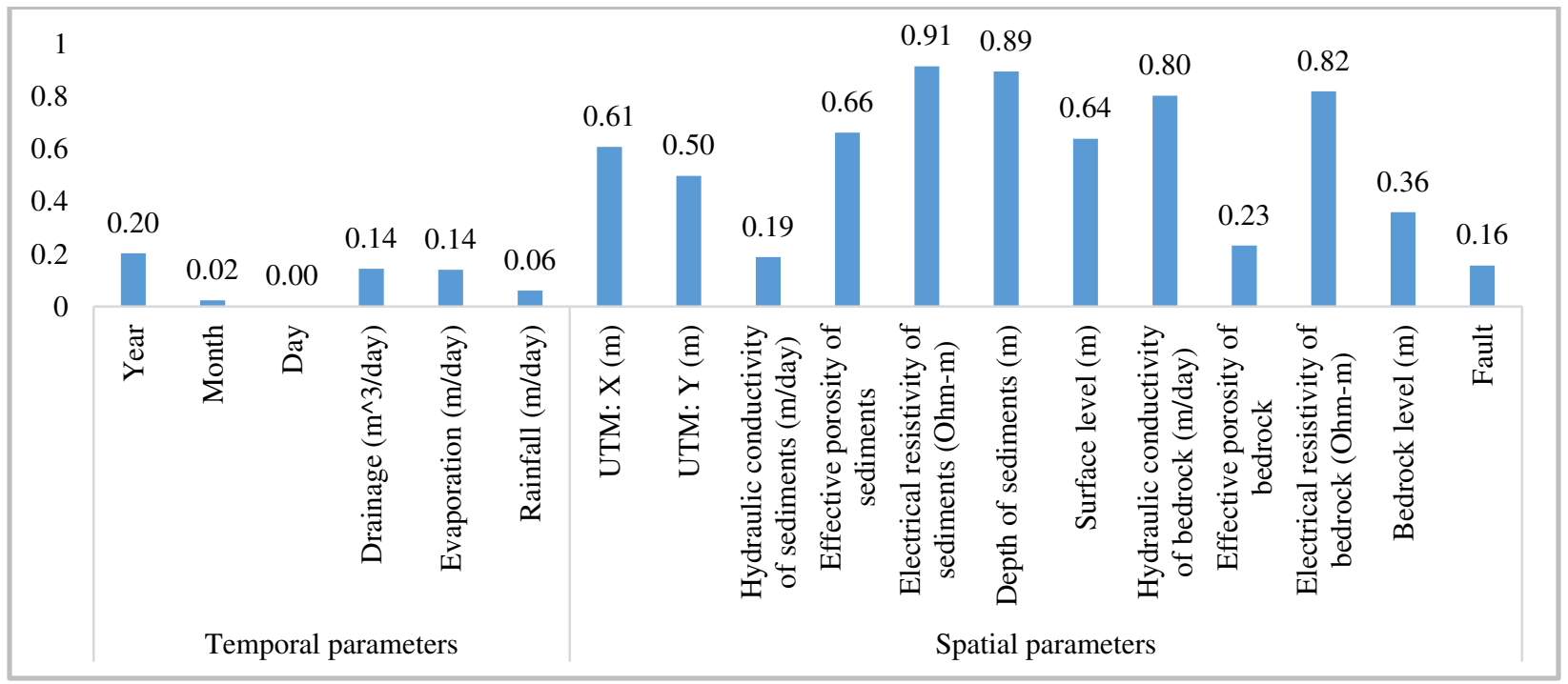

Fig. 14. Relevancy factor for temporal and spatial parameters on the groundwater level. 


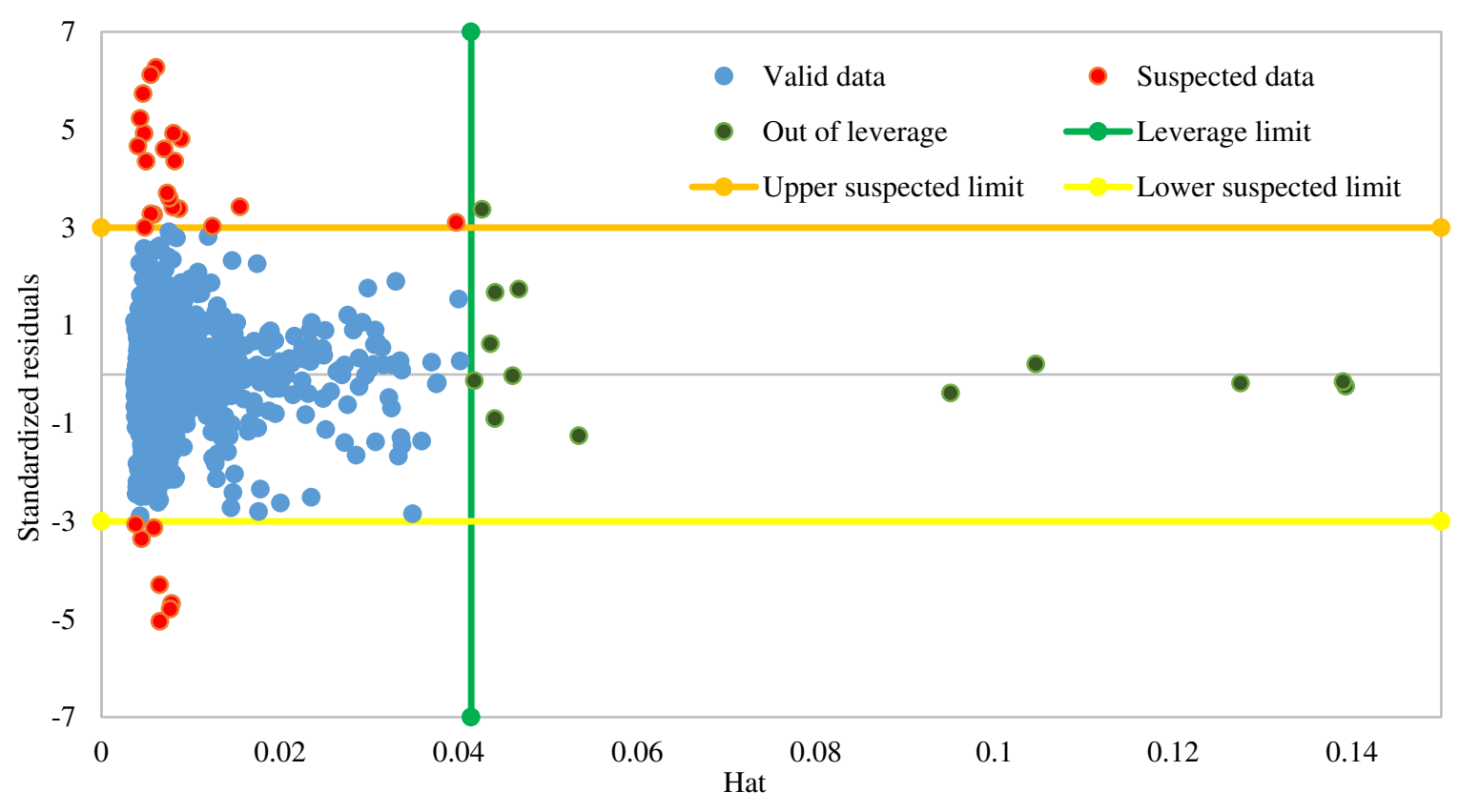

623

Fig. 15. William's plot for the resulting outputs by the proposed CMIS model. 


\begin{tabular}{|c|c|c|c|c|c|c|c|c|c|c|c|c|}
\hline \multicolumn{13}{|c|}{$\begin{array}{l}\text { Table } 1 \\
\text { Spatial input parameters of the well piezometer over the Gohar Zamin Iron Ore Mine }\end{array}$} \\
\hline $\begin{array}{c}\text { Number of } \\
\text { well } \\
\text { piezometers }\end{array}$ & $\begin{array}{l}\text { UTM:X } \\
\text { (m) }\end{array}$ & $\begin{array}{l}\text { UTM:Y } \\
\text { (m) }\end{array}$ & $\begin{array}{c}\text { Hydraulic } \\
\text { conductivity } \\
\text { of sediments } \\
\text { (m/day) }\end{array}$ & $\begin{array}{c}\text { Effective } \\
\text { porosity } \\
\text { of } \\
\text { sediments }\end{array}$ & $\begin{array}{c}\text { Electrical } \\
\text { resistivity } \\
\text { of } \\
\text { sediments } \\
(\text { Ohm-m) }\end{array}$ & $\begin{array}{c}\text { Depth of } \\
\text { sediments } \\
\text { (m) }\end{array}$ & $\begin{array}{c}\text { Surface } \\
\text { level } \\
\text { (m) }\end{array}$ & $\begin{array}{c}\text { Hydraulic } \\
\text { conductivity } \\
\text { of bedrock } \\
\text { (m/day) }\end{array}$ & $\begin{array}{c}\text { Effective } \\
\text { porosity } \\
\text { of } \\
\text { bedrock }\end{array}$ & $\begin{array}{c}\text { Electrical } \\
\text { resistivity } \\
\text { of } \\
\text { bedrock } \\
\text { (Ohm-m) }\end{array}$ & $\begin{array}{c}\text { Bedrock } \\
\text { level } \\
(\mathrm{m})\end{array}$ & Fault \\
\hline 1 & 333,513 & $3,219,470$ & 200 & 15 & 14.9 & 130.55 & 1724.47 & 19 & 0.09 & 31.6 & 1590.37 & $x$ \\
\hline 2 & 332,489 & $3,219,947$ & 187 & 20 & 29.4 & 180 & 1735.77 & 20 & 0.07 & 17.2 & 1645.77 & $x$ \\
\hline 3 & 332,149 & $3,220,733$ & 150 & 18 & 55.8 & 212 & 1747.5 & 22 & 0.05 & 5.58 & 1641.5 & $x$ \\
\hline 4 & 332,861 & $3,221,499$ & 160 & 14 & 9.69 & 139 & 1733.47 & 19 & 0.06 & 36.5 & 1663.97 & $x$ \\
\hline 5 & 334,438 & $3,220,764$ & 315 & 10 & 22.3 & 141.65 & 1729.92 & 17 & 0.1 & 13.5 & 1659.1 & $\checkmark$ \\
\hline
\end{tabular}




\section{Table 2}

The statistical explanation of the temporal input parameters

\begin{tabular}{|c|c|c|c|}
\hline & Drainage $\left(\mathrm{m}^{3} /\right.$ day) & Evaporation (m/day) & Rainfall (m/day) \\
\hline Mean & 9366 & 0.007 & 0.02 \\
\hline Median & 9183 & 0.006 & 0.006 \\
\hline Mode & 9504 & 0.004 & 0 \\
\hline Skewness & 0.119 & 0.257 & 0.862 \\
\hline Kurtosis & 0.245 & -1.345 & 0 \\
\hline Minimum & 0 & 0.002 & 0.09 \\
\hline Maximum & 17466 & 0.013 & \\
\hline
\end{tabular}




\section{Table 3}

The statistical parameters of the developed models for prediction of groundwater level using 1377 data points

\begin{tabular}{|c|c|c|c|c|c|c|c|c|c|c|c|c|c|}
\hline \multirow{2}{*}{$\begin{array}{l}\text { Statistical } \\
\text { parameters }\end{array}$} & \multicolumn{3}{|c|}{$\operatorname{ARE}(\%)$} & \multicolumn{3}{|c|}{$\operatorname{AARE}(\%)$} & \multicolumn{3}{|c|}{ SD } & \multicolumn{3}{|c|}{ RMSE } & \multirow{2}{*}{$\begin{array}{c}\mathrm{T}(\mathrm{s}) \\
\text { One } \\
\text { iteration }\end{array}$} \\
\hline & $\begin{array}{c}\text { Training } \\
\text { data }\end{array}$ & $\begin{array}{l}\text { Test } \\
\text { data }\end{array}$ & Total & $\begin{array}{c}\text { Training } \\
\text { data }\end{array}$ & $\begin{array}{l}\text { Test } \\
\text { data } \\
\end{array}$ & Total & $\begin{array}{c}\text { Training } \\
\text { data }\end{array}$ & $\begin{array}{l}\text { Test } \\
\text { data }\end{array}$ & Total & $\begin{array}{c}\text { Training } \\
\text { data }\end{array}$ & $\begin{array}{l}\text { Test } \\
\text { data } \\
\end{array}$ & Total & \\
\hline MLP-LM & -0.00014 & 0.00077 & -0.00095 & 0.01678 & 0.01961 & 0.01751 & 0.00026 & 0.00028 & 0.00027 & 0.43486 & 0.46825 & 0.44701 & 39.8 \\
\hline MLP-SCG & 0.00013 & 0.00372 & -0.0001 & 0.03514 & 0.0389 & 0.03522 & 0.00052 & 0.00053 & 0.00051 & 0.85940 & 0.86466 & 0.84850 & 16.32 \\
\hline MLP-RB & -0.00006 & 0.00265 & 0.00046 & 0.01872 & 0.02402 & 0.01992 & 0.00028 & 0.00037 & 0.00030 & 0.46062 & 0.60489 & 0.49080 & 14.36 \\
\hline CF-LM & 0.00188 & 0.00609 & 0.00212 & 0.02053 & 0.02591 & 0.02123 & 0.00031 & 0.00040 & 0.00032 & 0.50853 & 0.65183 & 0.52822 & 15.5 \\
\hline CF-BR & 0.00046 & -0.00052 & 0.00042 & 0.01741 & 0.02016 & 0.01791 & 0.00026 & 0.00029 & 0.00027 & 0.43614 & 0.48447 & 0.44753 & 16.41 \\
\hline CF-SCG & -0.00017 & -0.00414 & -0.00076 & 0.03098 & 0.03671 & 0.03153 & 0.00045 & 0.00052 & 0.00045 & 0.73403 & 0.86044 & 0.74641 & 13.88 \\
\hline CF-RB & -0.00001 & 0.00474 & 0.00083 & 0.02404 & 0.02384 & 0.02419 & 0.00036 & 0.00031 & 0.00036 & 0.59830 & 0.52017 & 0.59196 & 17.98 \\
\hline $\mathrm{RBF}$ & 0.00006 & -0.0182 & -0.00266 & 0.04173 & 0.04892 & 0.04322 & 0.00055 & 0.00063 & 0.00057 & 0.90080 & 1.03346 & 0.93469 & 9.58 \\
\hline GR & 0.00077 & 0.004 & 0.00035 & 0.0352 & 0.03805 & 0.03557 & 0.00052 & 0.00054 & 0.00053 & 0.85575 & 0.88188 & 0.86330 & 7.92 \\
\hline
\end{tabular}




\begin{tabular}{|c|c|c|c|c|c|c|}
\hline \multicolumn{7}{|c|}{$\begin{array}{l}\text { Table } 4 \\
\text { The statistical parameters an } \\
\text { extra validation data points }\end{array}$} \\
\hline Models & $\begin{array}{l}\text { ARE } \\
(\%)\end{array}$ & $\operatorname{AARE}(\%)$ & SD & RMSE & Function used & $\begin{array}{c}\text { Best } \\
\text { architecture }\end{array}$ \\
\hline MLP-LM & -0.12983 & 0.16232 & 0.00177 & 2.77969 & Logsig-Purelin-Purelin & $18-12-8-1$ \\
\hline MLP-BR & -0.37058 & 0.37081 & 0.00396 & 6.20605 & Elliot2sig-Purelin-Purelin & $18-12-8-1$ \\
\hline MLP-SCG & -0.08426 & 0.20241 & 0.00230 & 3.60198 & Elliot2sig-Purelin-Purelin & $18-12-8-1$ \\
\hline MLP-RB & -0.30413 & 0.30413 & 0.00338 & 5.29602 & Elliot2sig-Purelin-Purelin & $18-12-8-1$ \\
\hline CF-LM & -0.55160 & 0.55160 & 0.00569 & 8.90091 & Elliot2sig-Purelin-Purelin & $18-6-20-12-1$ \\
\hline CF-BR & -0.08841 & 0.13097 & 0.00160 & 2.50210 & Tansig-Logsig-Purelin & $\begin{array}{c}\text { 18-10-20-12- } \\
1\end{array}$ \\
\hline CF-SCG & -0.14831 & 0.17299 & 0.00195 & 3.05459 & Tansig-Logsig-Purelin & $18-8-20-12-1$ \\
\hline CF-RB & 0.16633 & 0.18931 & 0.00217 & 3.40278 & Tansig-Logsig-Purelin & $18-8-11-7-1$ \\
\hline $\mathrm{RBF}$ & -0.12491 & 0.12945 & 0.00157 & 2.46439 & Gaussian & $18-30-1$ \\
\hline GR & -0.84633 & 0.84632 & 0.00873 & 13.6627 & Gaussian & - \\
\hline CMIS & -0.10723 & 0.11340 & 0.00141 & 2.22181 & - & - \\
\hline
\end{tabular}




\section{Figures}
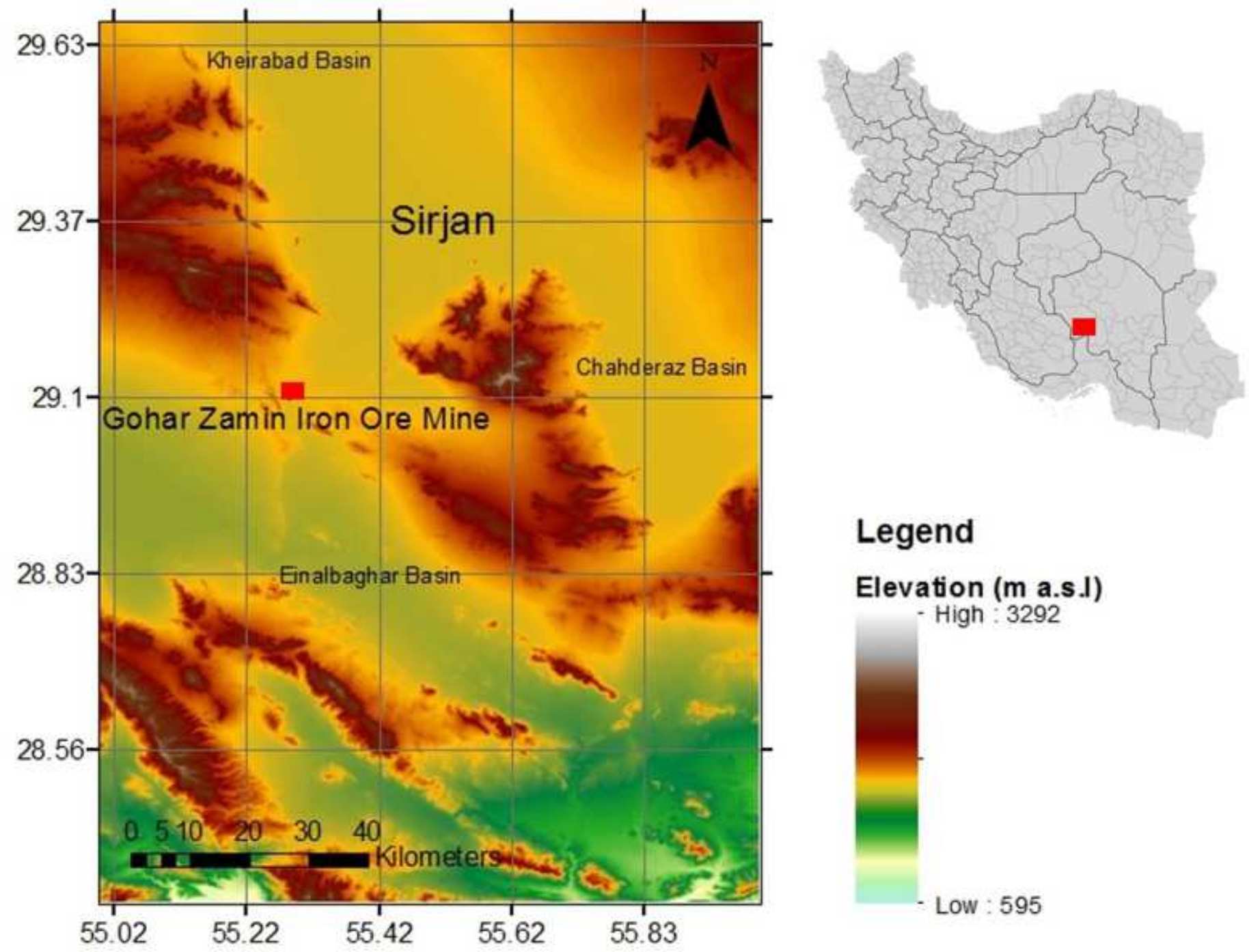

\section{Legend}

Elevation ( $m$ a.s.l)

- High : 3292

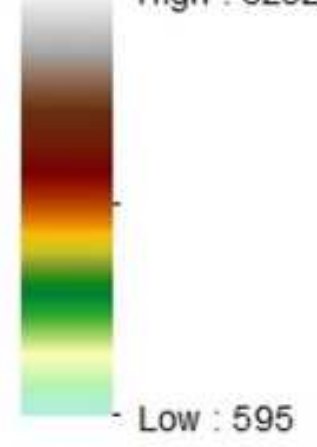

Figure 1

Study area and location of Gohar-Zamin Iron Ore Mine. Note: The designations employed and the presentation of the material on this map do not imply the expression of any opinion whatsoever on the part of Research Square concerning the legal status of any country, territory, city or area or of its authorities, or concerning the delimitation of its frontiers or boundaries. This map has been provided by the authors. 


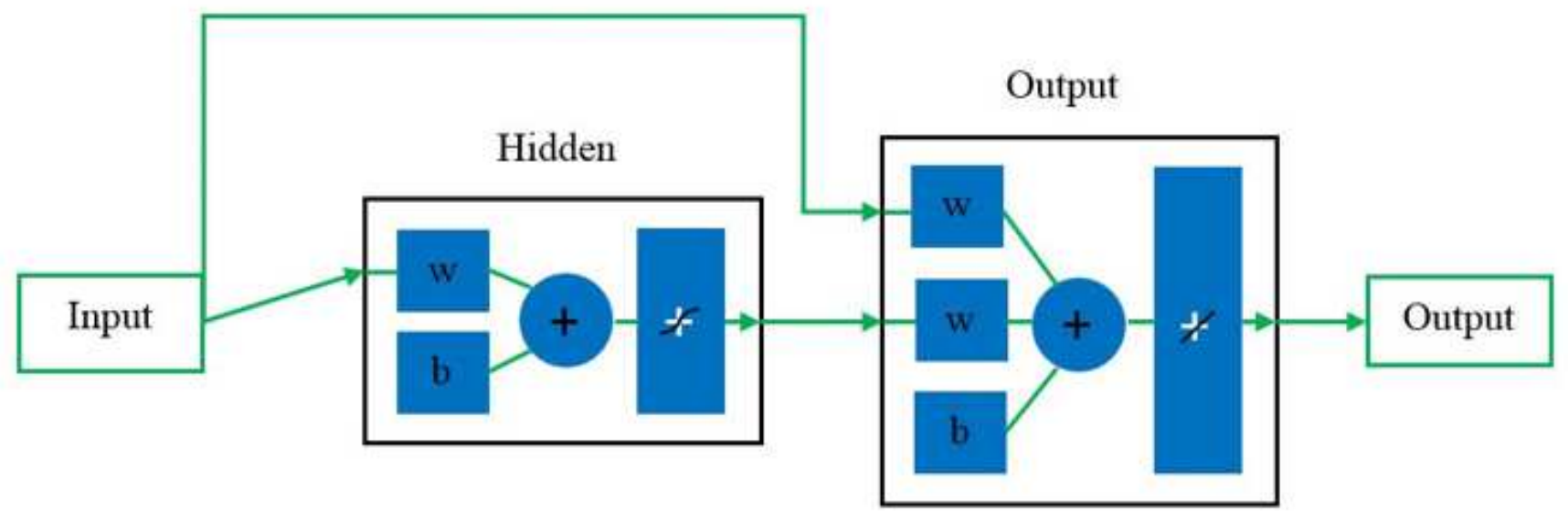

Figure 2

The architecture of CF neural network.

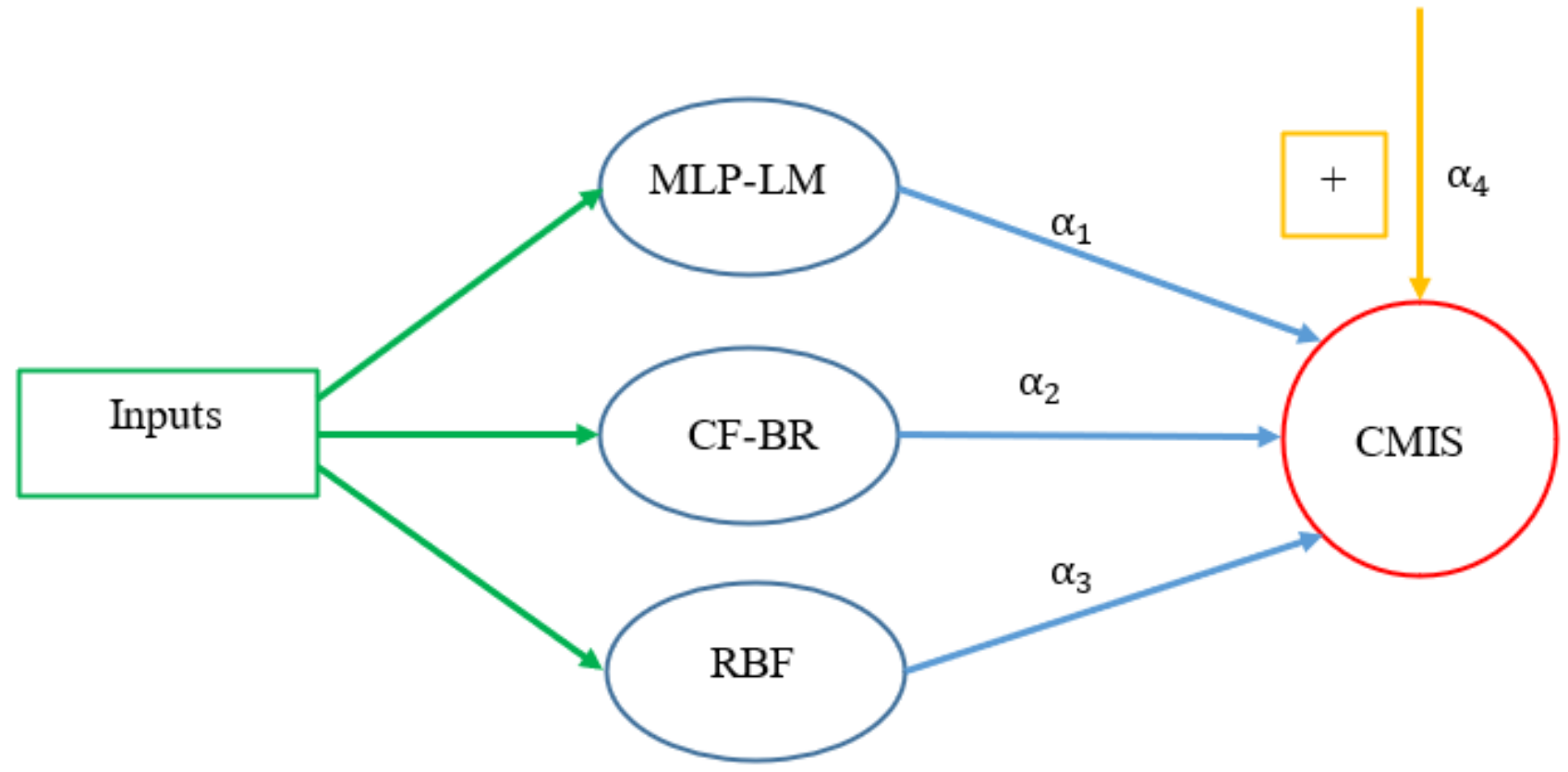

Figure 3

The architecture of the proposed CMIS model. 


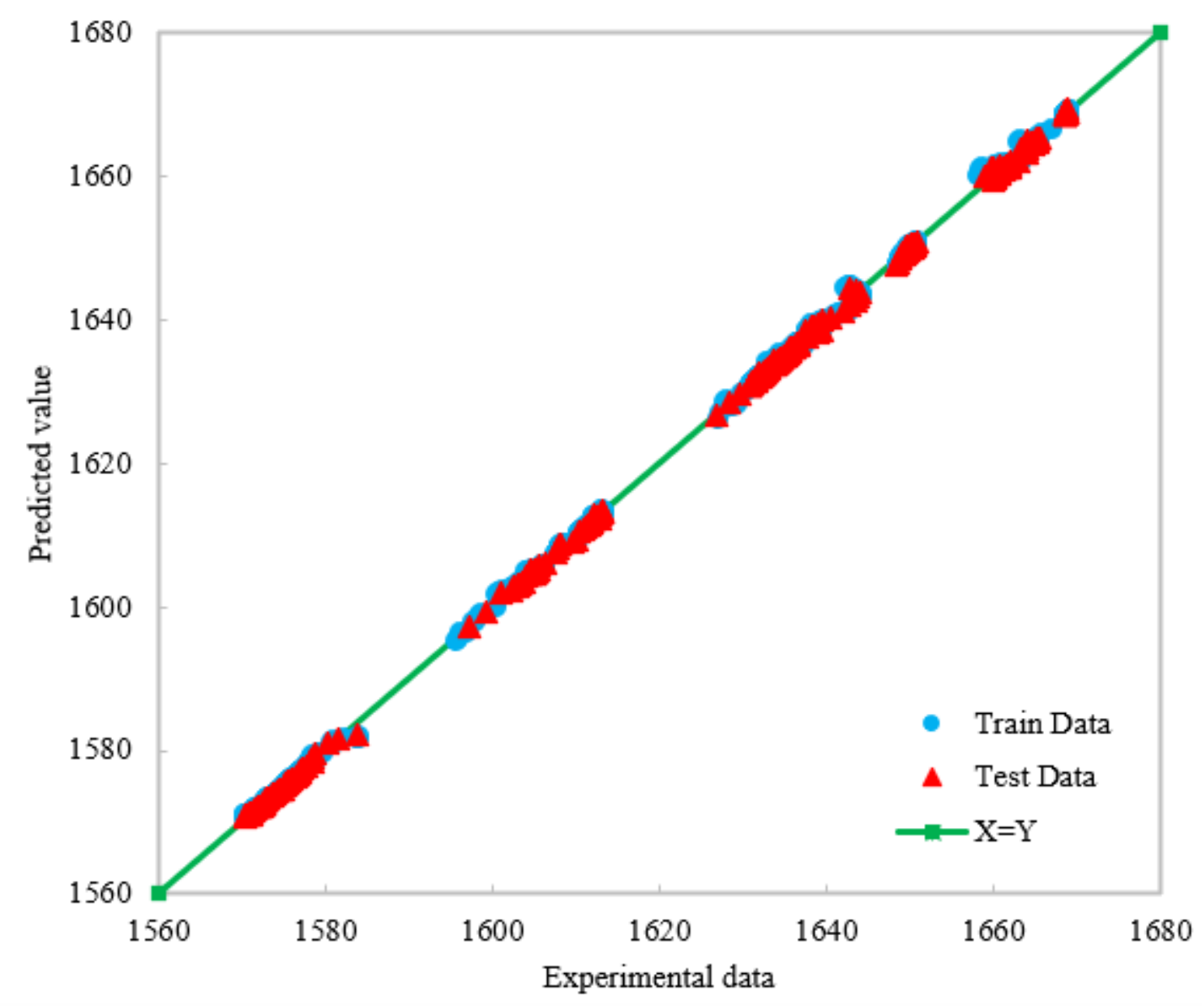

Figure 4

Cross plot of predicted relative groundwater level versus tentative relative groundwater level for test and train data using 1377 data points. 


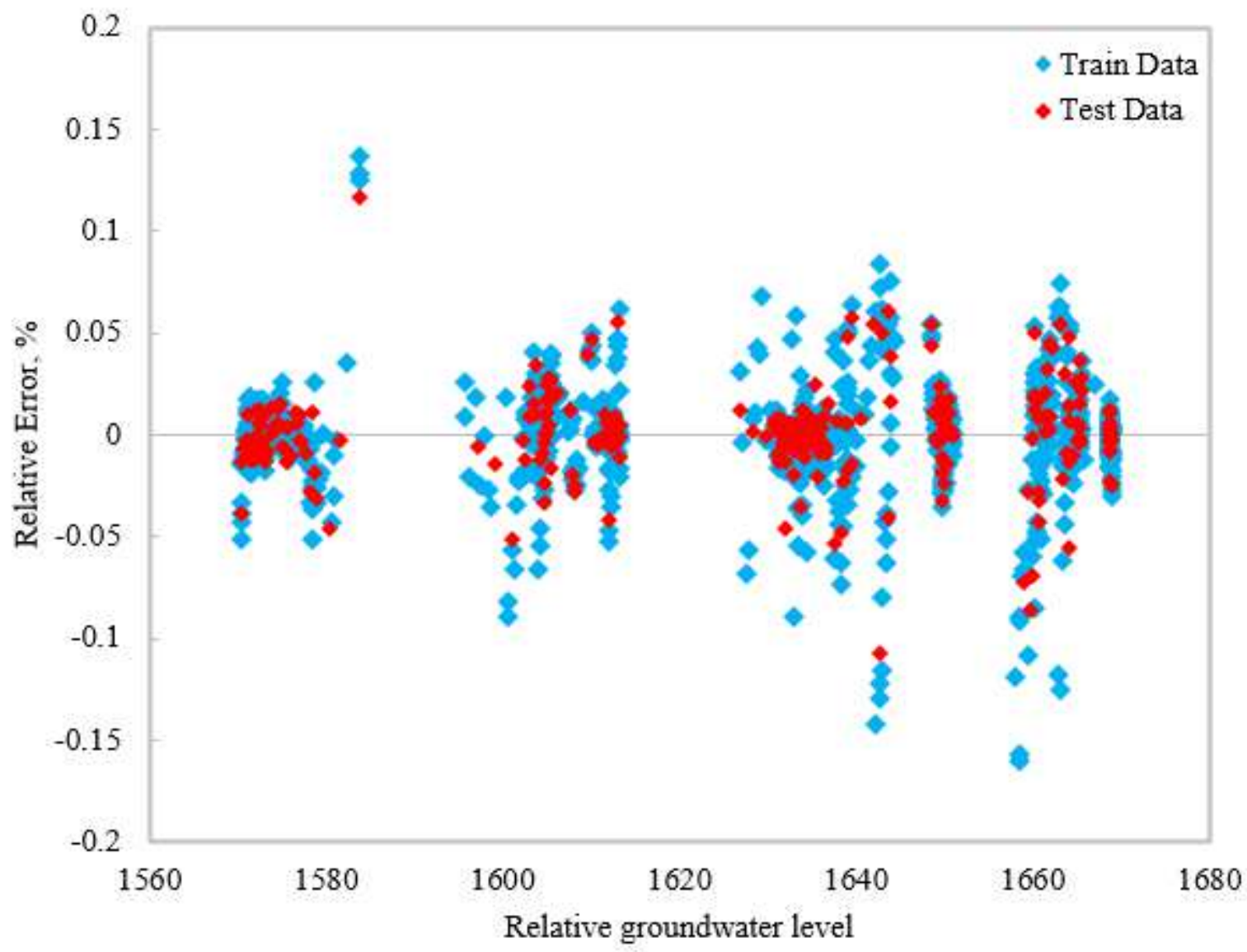

Figure 5

The relative error between the tentative and predicted relative groundwater level versus tentative relative groundwater level for train and test data using 1377 data points.

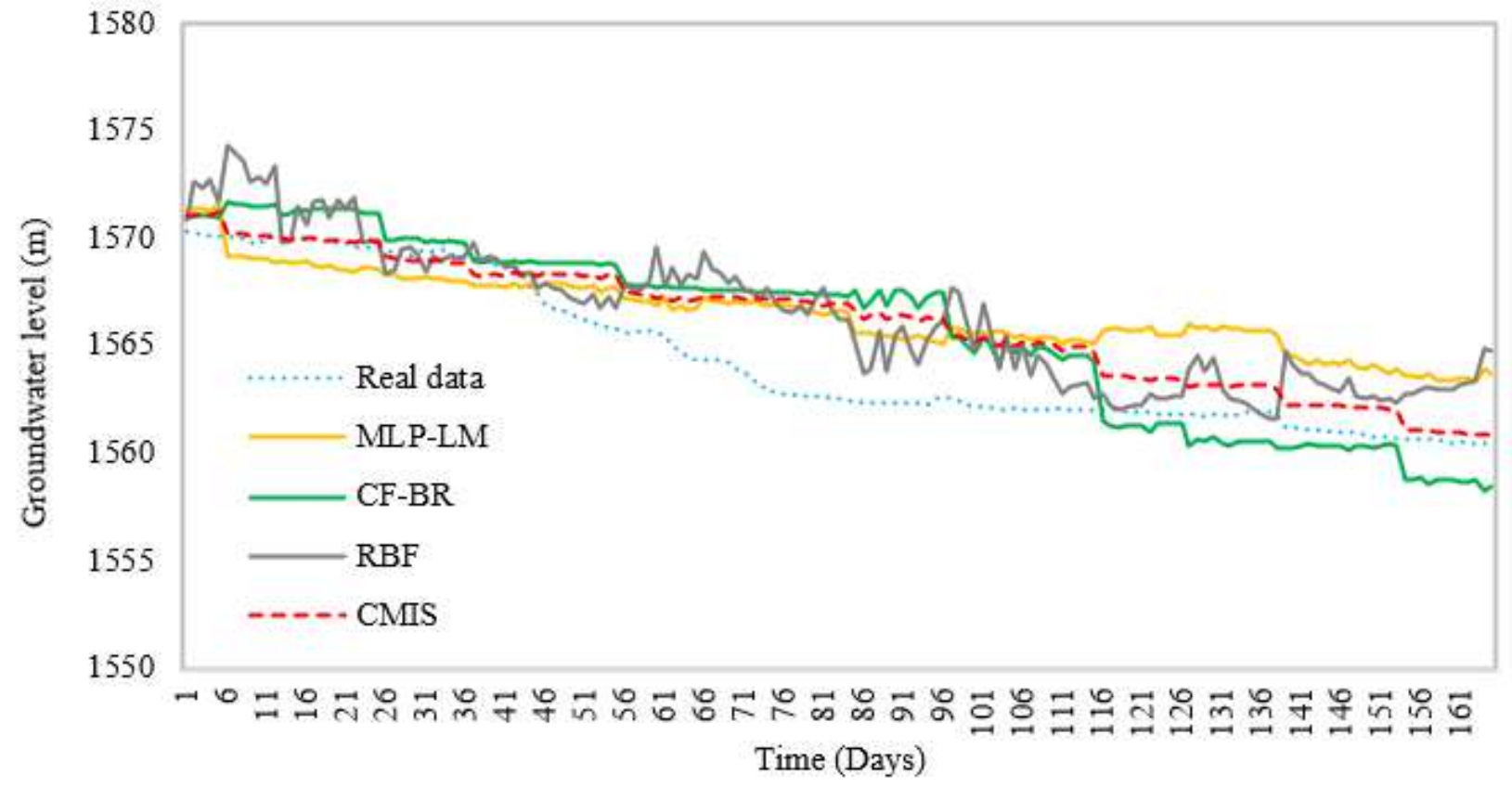


Figure 6

Prediction of groundwater level for different models using 165 extra data points.

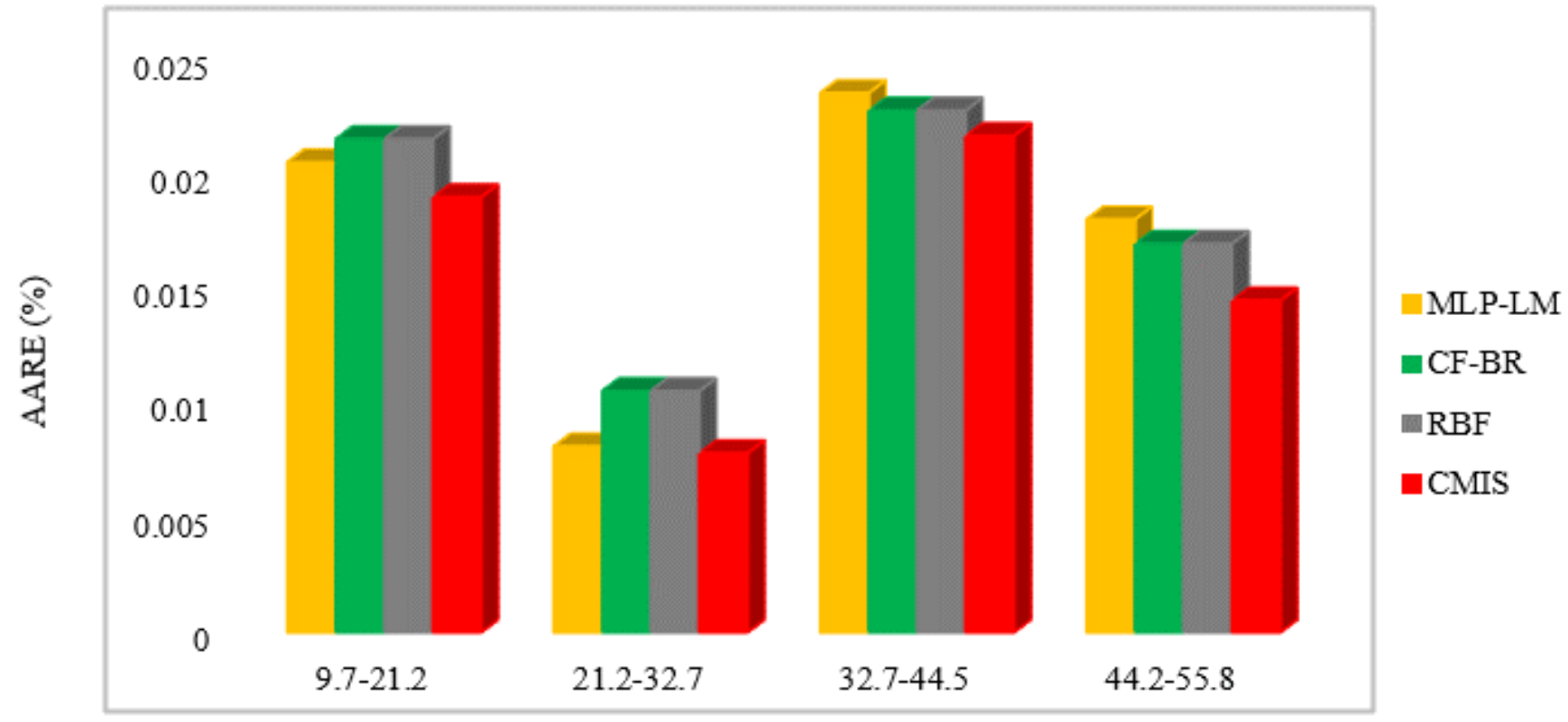

Electrical resistivity of sediments (ohm-m)

\section{Figure 7}

AARE (\%) for the developed CMIS model and best models for different electrical resistivity of sediments ranges.

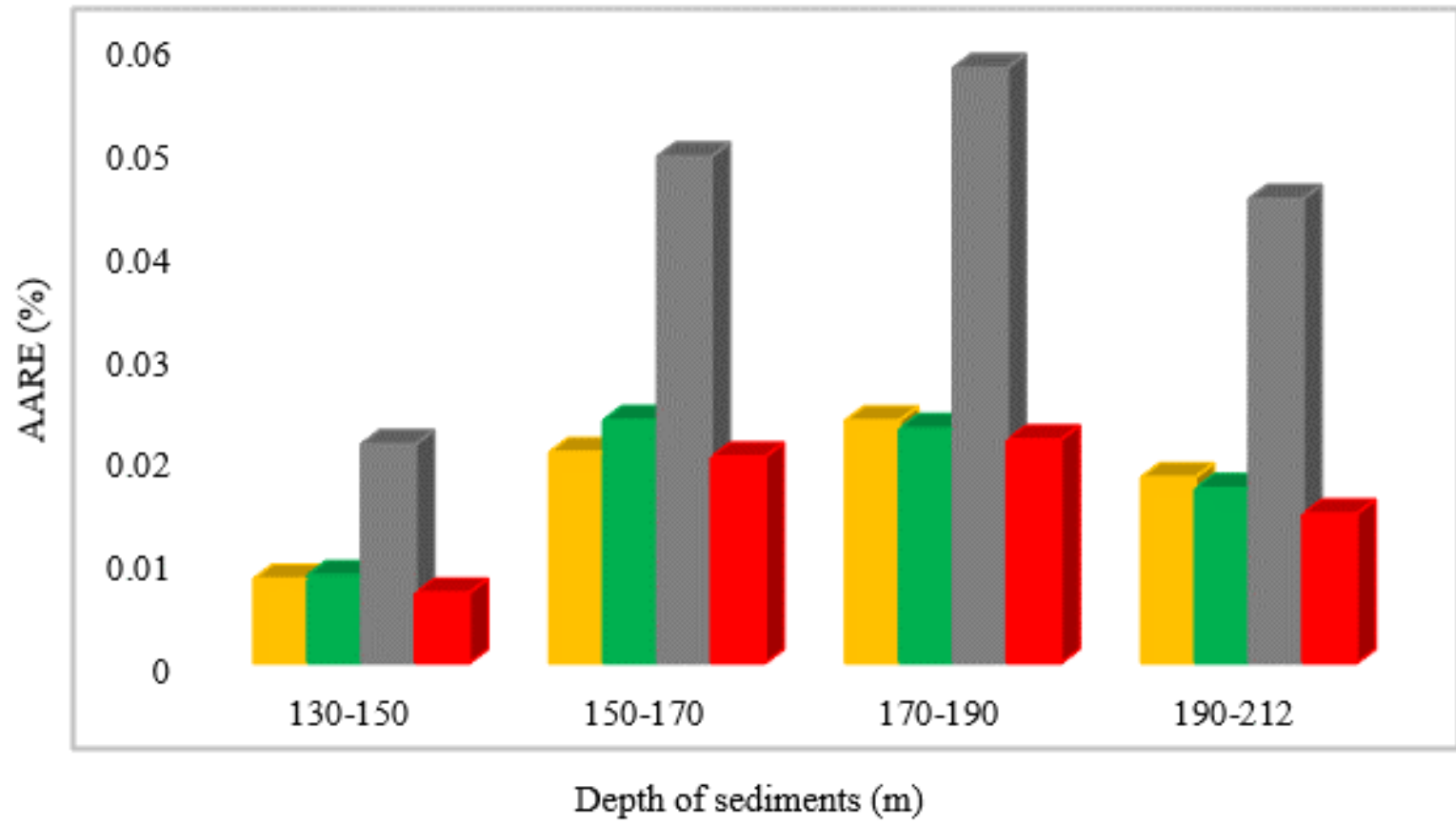

MLP-LM

- CF-BR

$\mathrm{RBF}$

- CMIS 
AARE (\%) for the developed CMIS model and best models for the depth of sediments ranges.

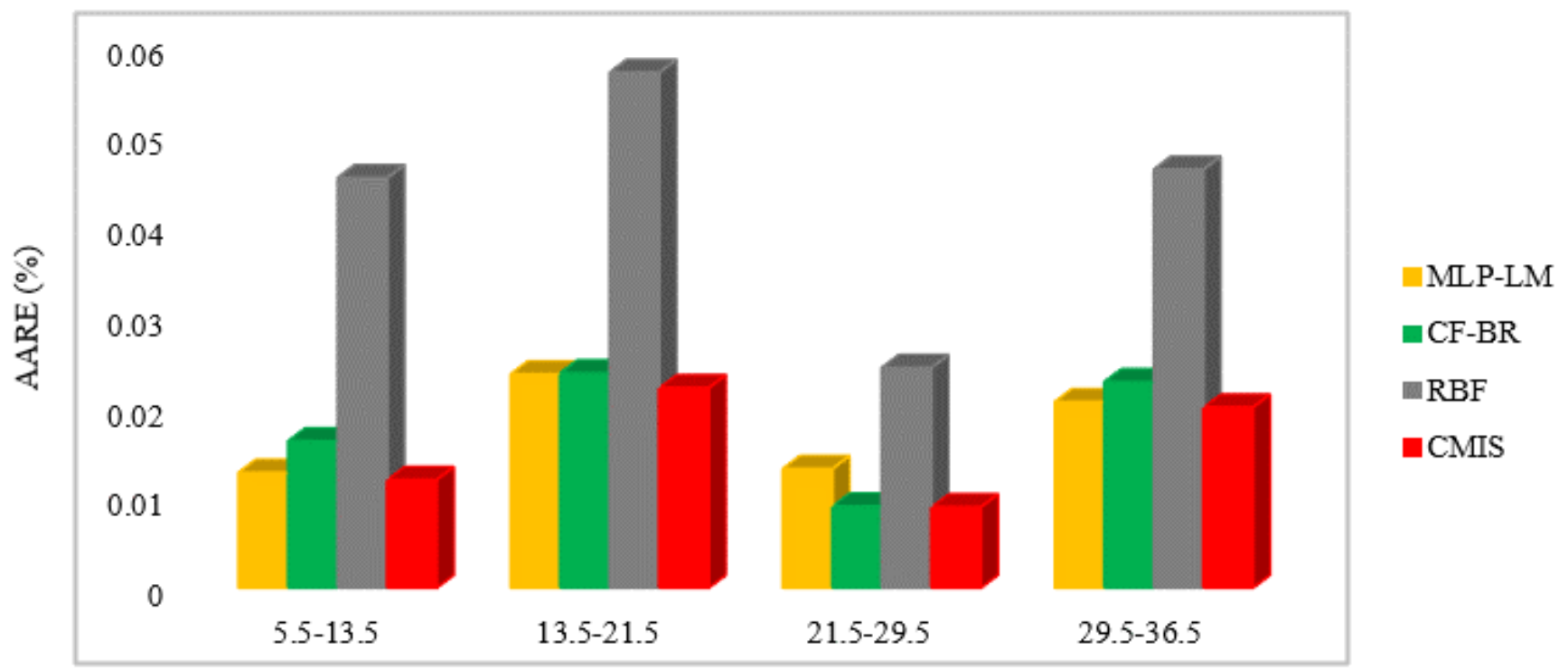

Electrical resistivity of bedrock (ohm-m)

\section{Figure 9}

AARE (\%) for the developed CMIS model and best models for electrical resistivity of bedrock ranges.

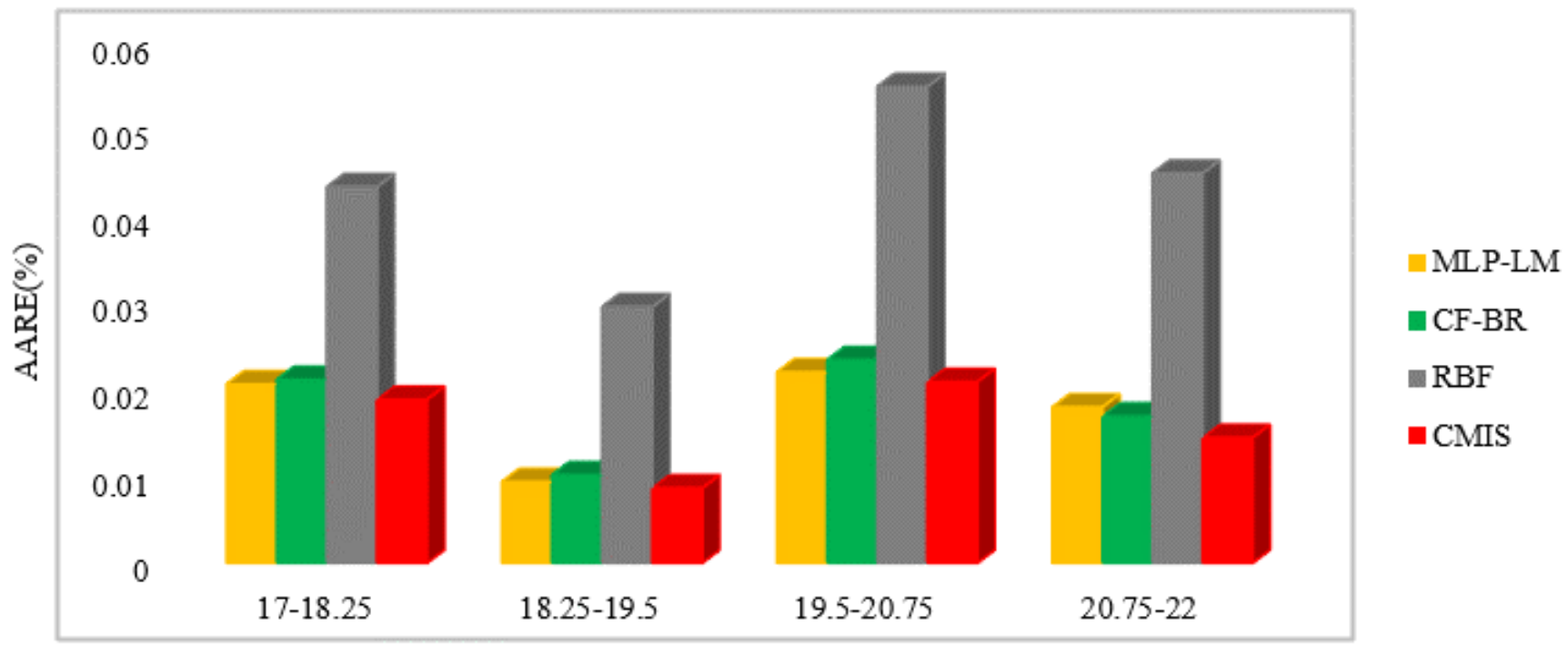

Hydraulic conductivity of bedrock ( $\mathrm{m} /$ day)

Figure 10

AARE (\%) for the developed CMIS model and best models for hydraulic conductivity of bedrock ranges. 


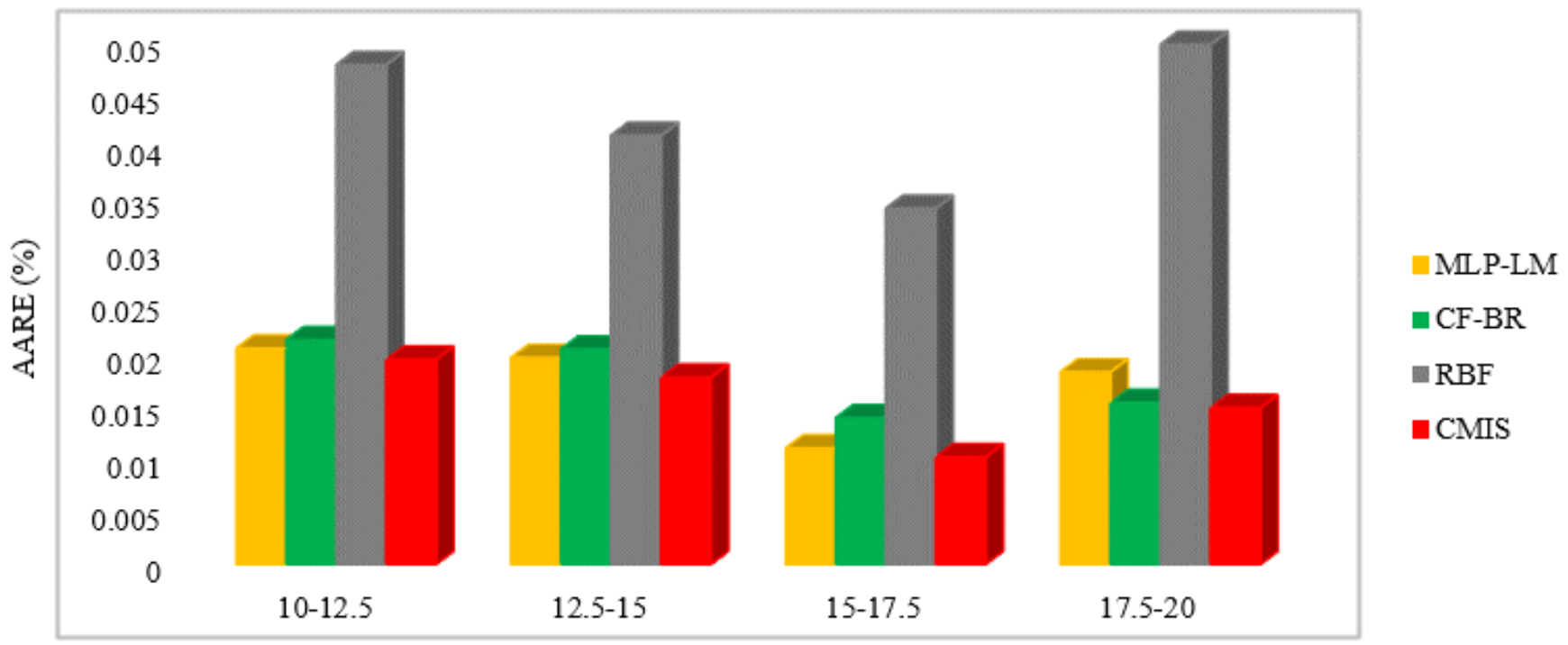

Effective porosity of sediments

\section{Figure 11}

AARE (\%) for the developed CMIS model and best models for effective porosity of sediments ranges.

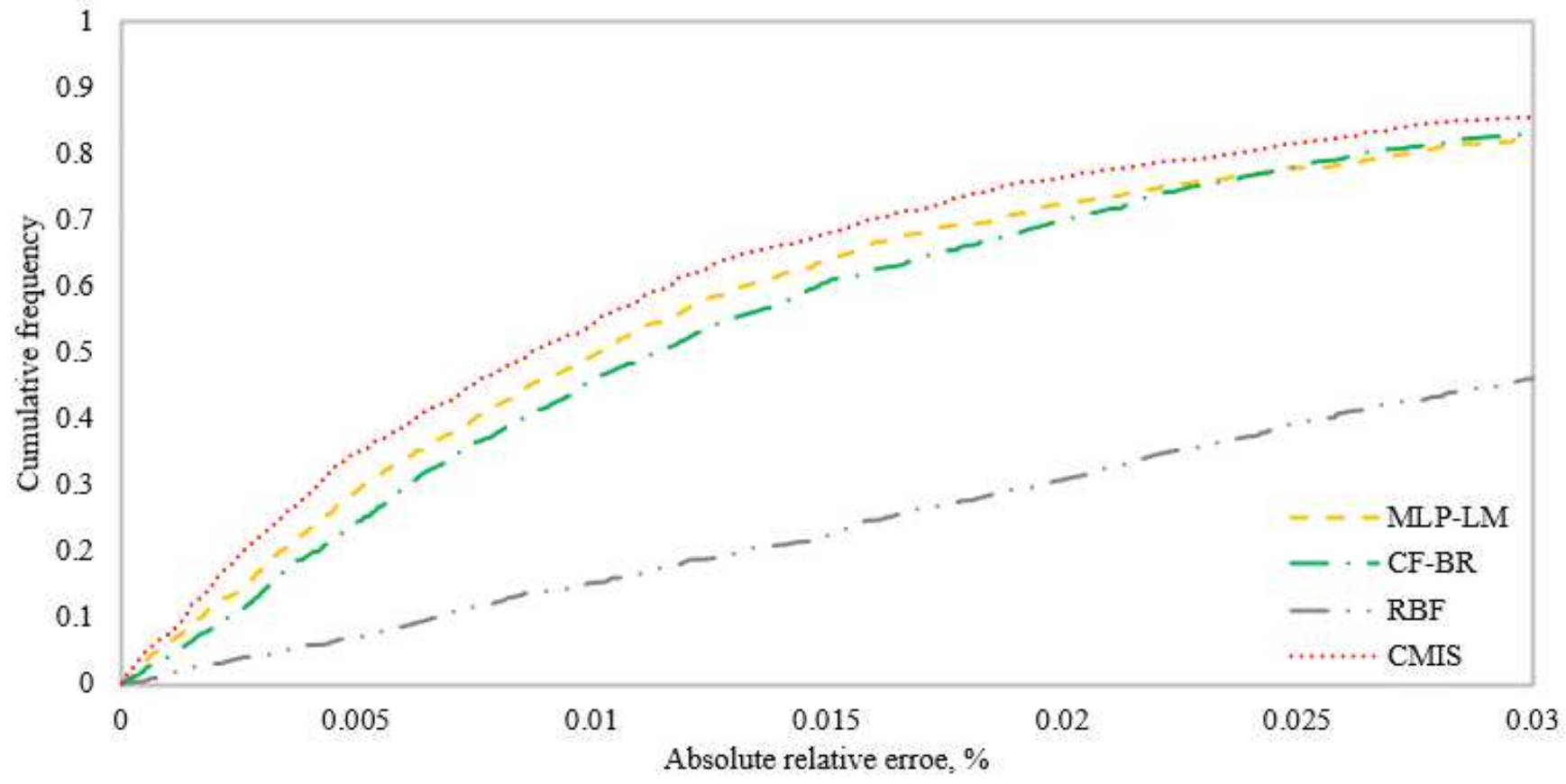

Figure 12

Cumulative frequency versus absolute relative error for all developed models. 


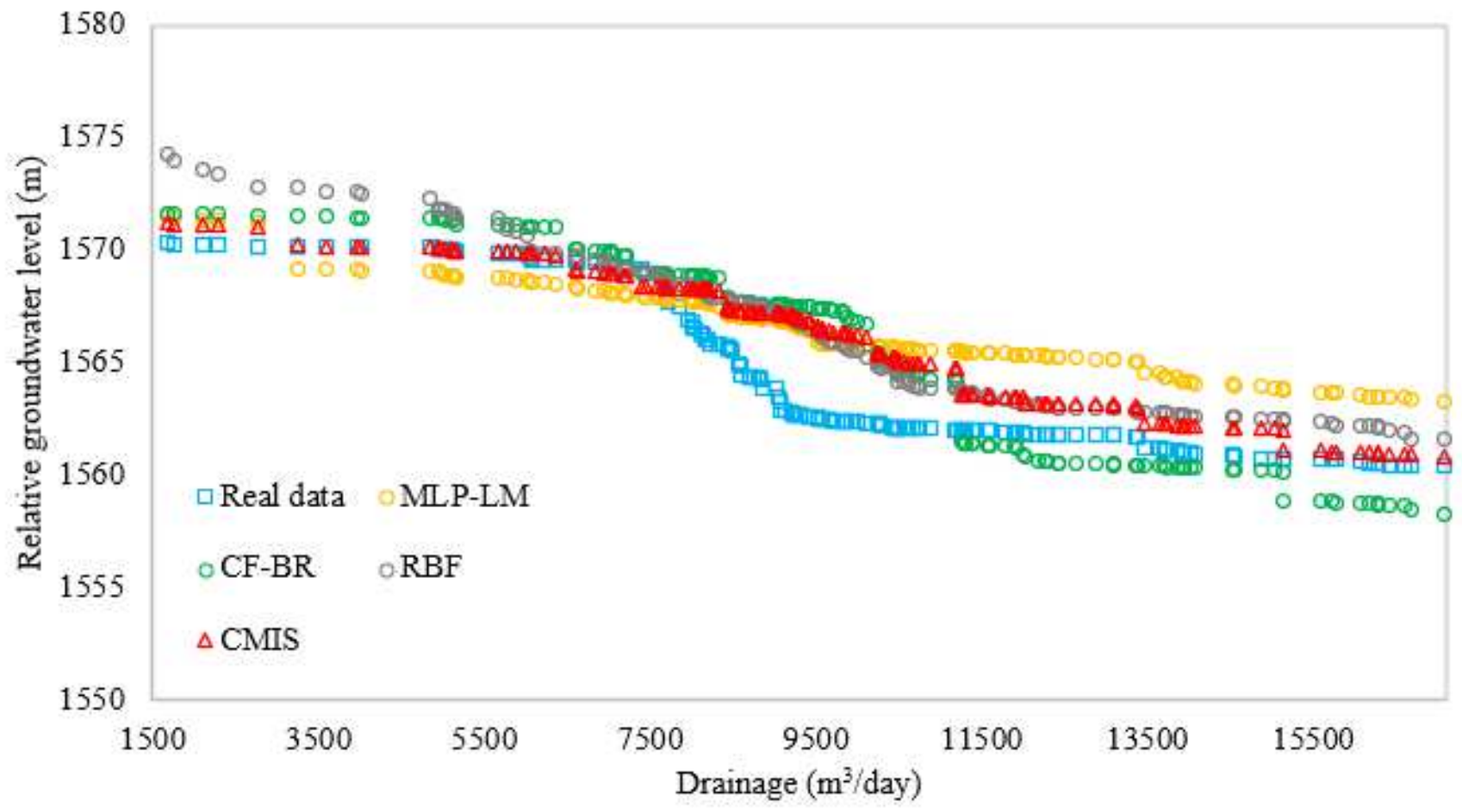

\section{Figure 13}

Comparing the relative variation with drainage for the proposed CMIS model and other developed models with real data.

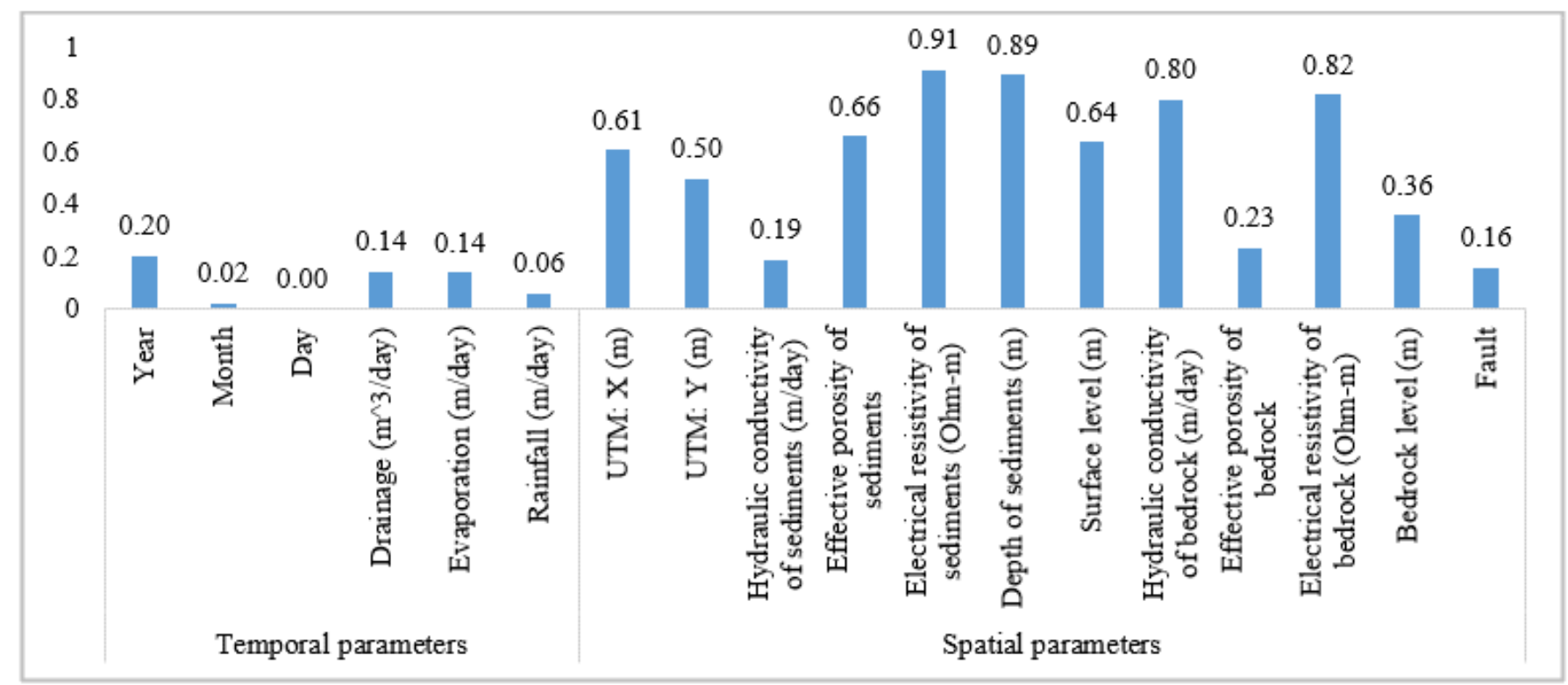

Figure 14

Relevancy factor for temporal and spatial parameters on the groundwater level. 


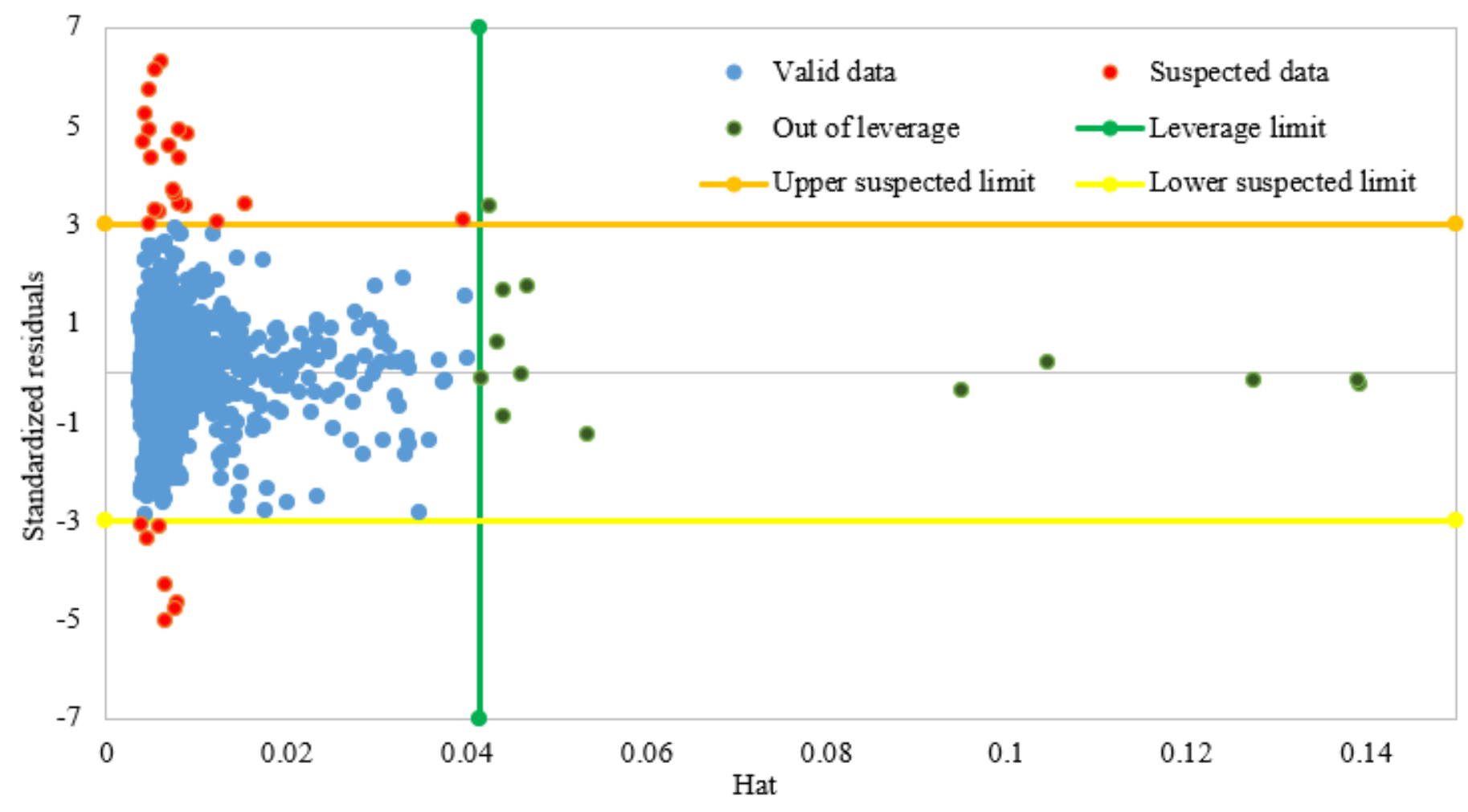

Figure 15

William's plot for the resulting outputs by the proposed CMIS model. 